[Aus dem hygienischen Institut der Universität Breslau.]

\title{
Ueber den Einfluss \\ des Windes auf die Wärmeabgabe todter Objecte.
}

$$
\text { Von }
$$

\section{Dr. Bruno Heymann,}

Assisteuten am Institut.

Dass die Bewegung der uns umgebenden Luft einen wesentlichen Einfluss auf unsere Körperwärme ausübt, ist eine längst bekannte, geradezu rolksthümliche Erfahrungsthatsache: Die angenehm kühlende Wirkung bewegter Fächer oder Tücher, die belebende Kraft eines Luftzuges an schwülen Sommertagen sind Jedermann ebenso geläufig wie die unerwünschte Steigerung des Kältegefühls durch heftigen Wind im Winter oder die vielgefürchtete schnelle Ablühlung einer umschriebenen Körperstelle durch "Zugluft". Auch der rasche Eintritt allgemeiner starker Kälteempfindung bei schneller Fortbewegung, z. B. bei rascher Fahrt in ungeschlossenem Wagen oder auf dem Deck eines Dampfers, weisen unzweifelhaft darauf hin, dass unser Körper bei raschem Wechsel der uns umgebenden Luft eine weit stärkere Entwärmung erleidet als in ruhiger oder wenig bewegter Luft.

Diese Beobachtungen unter den relativ engbegrenzten Witterungsschwankungen unserer Zone werden in interessanter Weise durch Reiseberichte aus Gegenden mit extremen klimatischen Verhältnissen ergänzt. So berichtet Adolf Erman und Payer, dass selbst in den Polargegenden Sibiriens die höchsten Kältegrade ohne Beschwerde zu ertragen wären, wenn keine stärkeren Winde herrschten, und andererseits erklärt Blanford ${ }^{1}$ in seinen "Climates and Weather of India:", dass die Fabrt durch

1 Citirt nach van Bebber, Hygienische Meteorologie. Stuttgart 1895. 
Bruno Heymann: Einfluss D. Windes a. D. Wümmeabgabe U. s. w. 197

das rothe Meer bei $32^{\circ}$ und Windstille viel drückender und unerträglicher gewesen sei, als der Aufenthalt im nordwestlichen Indien bei Temperaturen von $44^{\circ}$ bis $48^{\circ}$ während des Wehens warmer Winde.

So werthroll nun aber zweifellos alle solehe Hinweise auf die entwärmende Wirkung der bewe g ten Luft auch sind, so gestatten sie gleichwohl keinerlei Schlüsse auf die Grösse dieses Einflusses im Wettstreit mit den anderen, gleichzeitig wirksamen, meteorologischen Factoren. Bei der grossen Mannigfaltigkeit derselben wird Seitens des Beobachters stets die Neigung bestehen, die wahrgenommenen, angenehmen oder unangenehmen Empfindungen lediglich auf das hervorstechendste Witterungsmoment zu beziehen, die anderen Factoren aber, wie den Wechsel der Luft- und Bodentemperaturen, der Luftfeuchtigkeit, der Sonnenstrahlung u.s.f., unberücksichtigt zu lassen.

Ein weit besserer Einblick in den ursächlichen Zusammenhang zwischen Windbewegung und Wärmeabgabe wird offenbar von Versuchen derart zu erwarten sein, dass unter Beibehaltung sonst völlig gleicher Bedingungen ausschliesslich die Luftbewegung in der Umgebung eines warmen Körpers geändert und seine jeweilige Wärmeabgabe bestimmt wird.

Zunächst liegt es nahe, solche Versuche an todten Objecten anzustellen. Solche physikalische Untersuchungen hat von bau- und gewerbetechnischen Gesichtspunkten aus schon vor Jahrzehnten Schinz ${ }^{1}$ in Angriff genommen und in seinem Werke "Die Wärme-Messkunst" berichtet. Seine Versuchsanordnung war folgende: Ein 3 Zoll hoher und 2 Zoll weiter Cylinder, in dessen $A x e$ ein Thermometer angebracht war, wurde mit warmem Wasser gefüllt und auf F'üssen in einen zweiten Cylinder von 4 Zoll Höhe und 3 Zoll Durchmesser gestellt, welcher durch eine centrale Düse am unteren Boden mit einem Gasometer in Verbindung stand. Vermittelst desselben konnte ein gemessenes Volum Luft pro Secunde durch den Apparat geführt und die Geschwindigkeit der dadurch erzeugten Luftströmung genau bestimmt werden. Mit Hülfe einer Secundenuhr wurden sodann auch die Zeiten notirt, die verflossen, bis das Thermometer um einen oder mehrere Grade sank. Da das Gewicht des Wassers in dem inneren Cylinder bekannt war, so ergab sich dann durch einfache Rechnung die transmittirte Wärmemenge.

Zur Erweiterung seiner Versuche benützte Schinz in der einen Versuchsreihe einen blanken Weissblech-Cylinder, in einer weiteren Versuchsrejhe denselben, aber mit Kienruss und Mohnöl übertünchten Cylinder.

${ }^{1}$ Schinz, Die Wärme-Messkunst. Stuttgart 1858. 
Da das Origiual nicht leicht zugänglich ist, so mügen die von Schinz gewonnenen Ergebnisse in der beigegebenen Tabelle I vollständig Platz finden. Leider ist die Beurtheilung derselben in Folge der gar zu knappen Darstellung der Versuchsbedingungen erschwert. Von ganz besonderer Bedeutung wäre die Angabe gewesen, ob für eine gründliche Durchmischung des Wassers in dem Abkühlungsobjecte Sorge getragen wurde. Des Weiteren rermisst man Angaben über die Dauer jedes Versuchs, über die absoluten Temperaturen der bewegten Luft und des Wassers, über die Lage der Thermometerkugel in dem letzteren u. a. m.

\section{Tabelle I.}

\section{Wärmeabgabe eines Metallcylinders in Windstille und Wind von verschiedener Stärke (nach Schinz).}

\begin{tabular}{|c|c|c|c|c|c|}
\hline \multicolumn{2}{|c|}{ Bei Luftströmungen } & $19^{\prime}$ pro Sec. & $2 \cdot 2^{\prime}$ pro Sec. & $1 \cdot 16^{\prime}$ pro Sec. & $\begin{array}{c}\text { Ohne } \\
\text { Strömung }\end{array}$ \\
\hline \multicolumn{2}{|c|}{$\begin{array}{l}\text { Temperaturdifferenz } \\
\text { zwischen Luft und } \\
\text { dem Wasser im Cylinder }\end{array}$} & $\begin{array}{l}\text { Wärme- } \\
\text { Einheiten }\end{array}$ & $\begin{array}{l}\text { Wärme- } \\
\text { Einheiten }\end{array}$ & $\begin{array}{l}\text { Wärme- } \\
\text { Einheiten }\end{array}$ & $\begin{array}{l}\text { Wärme- } \\
\text { Einheiten }\end{array}$ \\
\hline \multirow{7}{*}{$\begin{array}{c}\text { A. } \\
\text { Blanker } \\
\text { Cylinder } \\
\text { (Weissblech) }\end{array}$} & $67^{\circ}$ & 136 & 118 & 115 & 113 \\
\hline & 57 & 110 & 104 & 102 & 92 \\
\hline & 47 & 92 & 78 & 76 & 73 \\
\hline & 37 & 72 & 60 & 58 & 55 \\
\hline & 27 & 68 & 48 & 46 & 40 \\
\hline & 17 & 43 & 30 & 28 & 22 \\
\hline & 7 & 18 & 15 & 14 & 9 \\
\hline \multirow{7}{*}{$\begin{array}{c}\text { B. } \\
\text { Derselbe, mit } \\
\text { Kienruss und } \\
\text { Mohnöl } \\
\text { übertünchte } \\
\text { Cylinder }\end{array}$} & $67^{\circ}$ & 513 & 300 & 240 & 231 \\
\hline & 57 & 360 & 212 & 210 & 186 \\
\hline & +7 & 300 & 172 & 168 & 145 \\
\hline & 37 & 212 & 138 & 136 & 106 \\
\hline & 27 & 132 & 100 & 98 & 72 \\
\hline & 17 & 90 & 62 & 60 & 41 \\
\hline & 7 & 40 & 22 & 20 & 13 \\
\hline
\end{tabular}

Gleichwohl lassen sich, in Uebereinstimmung mit Schinz, aus der Tabelle zwei wichtige Thatsachen mit Sicherheit ableiten, nämlich erstens, dass bewegte Luft eine grössere Wärmeabgabe bewirkt als gleichwarme ruhende Luft, und zweitens, dass schnellere Luftströme die Wärmeabgabe, steigern, aber nicht proportional ihrer Geschwindigkeit, sondern mit stetig abnehmender Intensität. Fin mathematisch formulirtes Gesetz für diese Beziehungen wurde von Schinz nicht aufgestellt und ist, bei dem bereits berührten Mangel einiger wesentlichen Angaben, nachträglich nicht mehr herauszufinden. 
Eintluss des Windes aUf die Wärmeabgatie todtek ObJecte. 199

In Würdigung der grossen Bedentung, welche der Luftbewegung in hygienischer und praktisch-medicinischer Hinsicht zukommt, hat weiterhin Hiller ${ }^{1}$ einige diesbezügliche, physikalische Beobachtungen angestellt. Seine Versuchsanordnung war folgende: Eine mit warmem Wasser von $45^{\circ} \mathrm{C}$. gefüllte Glasflasche, durch deren Kautschukstopfen ein empfindliches Thermometer eingeführt war, wurde freischwebend im Zimmer aufgèhüngt und mittels einer kleinen, nach dem Takte eines Metronoms gedrehten, Handwindmühle Winden verschiedener Stärke (1,2,3 und $4^{\mathrm{m}}$ pro Sec.) jedes Mal so lange ausgesetzt, bis das Thermometer auf $30^{\circ}$ gesunken war.

Die Ergebnisse dieser Versuche stellen im Wesentlichen eine Bestätigung der bereits von Schinz beobachteten Erscheinungen dar.

Die Abkühlung der Glasflasche von $44^{\circ}$ auf $30^{\circ}$ erfolgte:

\section{Tabelle II.}

\begin{tabular}{|c|c|c|c|c|c|c|c|c|c|}
\hline & $W_{1 n}$ & ille & & & & und & $+17^{\circ} \mathrm{C}$ & 1 & 11 \\
\hline$"$ & Wind & ron & $1^{\mathrm{m}}$ & pro & Sec. & $"$ & $+19^{\circ} \mathrm{C}$ & $"$ & 72 \\
\hline 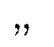 & $"$ & $"$ & $2^{\mathrm{m}}$ & $"$ & $"$ & $\varphi$ & $+13^{\circ} \mathrm{C}$ & $"$ & 44 \\
\hline & $"$ & $"$ & $3^{\mathrm{m}}$ & $"$ & $"$ & $"$ & $+17^{\circ} \mathrm{C}$ & $"$ & 37 \\
\hline & $"$ & "n & $4^{m}$ & $"$ & $"$ & $"$ & $+17^{\circ} \mathrm{C}$. & $"$ & \\
\hline
\end{tabular}

Auch hier springt zunächst der mächtige Einfluss der Luftbewegung auf die Würmeabgabe in die Augen: In Windstille erfolgt die Abkühlung in 116 Minuten; in Wind von nur $4 \mathrm{~m}$, wie er im Freien sehr häufig herrscht, in $291 / 2$ Minuten, also vier Mal so schnell. Des Weiteren zeigt sich auch hier und zwar mit noch grösserer Deutlichkeit wie in den Schinz'schen Versuchen die relatir grösste Wirkung bei den schwächeren Luftströmen, während bei den höheren Geschwindigkeiten zwar auch noch eine Beschleunigung der Abkühlung gegenüber den weniger schnellen zu Stande kommt, nicht aber proportional der Steigerung der Luftbewegung, sondern in einem weit geringeren, abnehmenden Maasse.

$\mathrm{Zu}$ ganz ähnlichen, nur in den absoluten Zahlen abweichenden Ergebnissen gelangte Hiller an der bekleideten oder stark angefeuchteten Glasflasche.

Bei seinen vornehmlich praktischen Gesichtspunkten hat Hiller keinen Versuch gemacht, die beobachteten Erscheinungen in ein Gesetz zusammenzufassen. Allerdings würde hierzu seine Versuchsanordnung auch kaum hingereicht haben. Der bedeutsamste Mangel derselben be-

1 Hiller, Ueber Erwärmung u. Abkühlung des Infanteristen auf dem Marsche und den Einfluss der Kleidung darauf. Deutsche militär-ärztliche Zeitschrift. 1885. XIV. Jabrg. S. 362 . 
steht darin, dass keinerlei Maassregel zur gründlichen Durchmischung der erkaltenden Wassermasse getroffen war. In Folge dessen mussten die kühleren Wassertheilchen nach unten sinken, die wärmeren die obere Hälfte der Kugel erfüllen und hierdurch die Gesammtwärmeabgabe, welche ja ausschliesslich von der Temperatur der Oberfläche abhängt, in uncontrolirbarer Weise beeinflusst werden. Aus demselben Grunde gestatten auch die Angaben des Thermometers keinen Rückschluss auf die Gesammtwärmeabgabe des Körpers, sondern nur auf die Temperaturerniedrigung einer Schicht.

Sodann herrscht bezüglich der zur Einwirkung gekommenen Luftströme insofern eine gewisse Unsicherheit, als erstens die Windgeschwindigkeit nicht mittels Anemometers in möglichster Nähe der Glasflasche bestimmt, sondern als Mittelwerth aus der Zahl der Umdrehungen und der Schaufelgrösse berechnet wurde, und zweitens der mühsame Handbetrieb der Mühle während der ganzen Versuchsdauer wohl kaum mit der erforderlichen Gleichmässigkeit durchzuführen war. Die Verwerthbarkeit der Beobachtungen leidet ferner darunter, dass die Differenzen zwischen der Anfangstemperatur der Kugel und der Lufttemperatur nicht in allen Versuchen übereinstimmen. Endlich ist auch ihre Anzahl und Variirung zu begrenzt, als dass die Ableitung gesetzmässiger Relationen möglich gewesen wäre.

Unbeabsichtigter, ja unerwünschter Weise hat weiterhin noch Pouillet Gelegenheit gehabt, im Verlaufe von Untersuchungen über die directe Sonnenstrahlung den entwärmenden Einfluss des Windes zu beobachten. Stellte er sein Pyrheliometer, einen mit Wasser gefüllten Kupfercylinder, im Freien ohne jeden Windschutz auf, so bemerkte er bei stärkerer Luftbewegung nicht unerhebliche Schwankungen in den Angaben seines Apparates. Zahlenmässige Belege für die Grösse dieses Einflusses finden sich in Cramer's Arbeit über "die Messung der Sonnenstrahlung in hygienischer Hinsicht". Cramer ${ }^{1}$ verglich die Angaben eines Pouillet'schen Pyrheliometers und eines Vacuumthermometers und konnte feststellen, dass das erstere bei gleicher Insolation im Wind wesentlich zu niedrige Angaben machte, während das Vacuumthermometer fast völlig unbeeinflusst blieb und daher eine directe Ablesung der durch die Luftberwegung bedingten Abweichung der Pyrheliometer-Angaben von dem wahren Werthe ermöglichte. In dem einen, hierüber angestellten Versuche ergab sich, dass bei einer Differenz der Sonnen- und Schattentemperatur von ca. $\mathbf{2 4}^{0}$ das Thermometer des Wassergefässes in unbewegter luft $3 \cdot 43^{\circ}$, in bewegter

- Cramer, Die Messung der Sonnenstrahlung in hygienischer Hinsicht. Archiv für Hygiene. Bd. XX. S. 313. 
Einfluss nes Windes auf die Wärmeabgabe todter Objecte. 201

Lutt von 1 bis $2^{\mathrm{m}}$ Geschwindigkeit nur $2.07^{\circ}$ bis $3 \cdot 15^{\circ}$ Erhöhung aufwies. -

Damit sind meines Wissens die bisherigen. Beobachtungen über erhöhte Wärmeabgabe todter Objecte im Winde erschöpft. Sie beweisen insgesammt den mächtigen Einfluss der Luftbewegung für die Entwärmung; sie lassen auch eine gewisse Gesetzmässigkeit zwischen dem Grade der Wärmeabgabe und der Windgeschwindigkeit vermuthen, haben jedoch ein exactes Gesetz hierfür nicht zu erbringen vermocht.

Auf Anregung von Herrn Geh.-Rath Flügge hat daher neuerdings von Schuckmann in den vorstehenden Versuchen die Frage wieder aufgenommen. Seine Versuchsanordnung hatte mit der oben erwähnten Hiller's grosse Aehnlichkeit.

Mit zwei tubusartigen Ansätzen versehene Blechcylinder oder Glaskugeln, welche mit warmem Wasser gefüllt waren, wurden mittels eines, durch ein Tretwerk in Bewegung gesetzten Ventilators Winden verschiedener Stärke ausgesetzt und die im Verlaufe von 5 Minuten erfolgende Abkühlung des Kugelinhaltes an einem, durch den einen Tubus eingeführten Thermometer gemessen. Die Durchmischung des Wassers wurde mittels einer, in dem anderen Tubus befestigten, kleinen leierartigen Kurbel vorgenommen, deren ins Wasser. ragendes Ende zwei schaufelartige Flügel trug. Die Tiurbel wurde nach $1 \frac{1}{2}, 3$ und $41 / 2$ Minuten Versuchsdaner je $1 /$, Minute lang in Bewegung gesetzt.

Als zusammenfassendes Lrgebniss seiner Versuche stellte von Schuckmann den Satz auf, dass ,die Wärmeabgabe, nach Abzug des auf Ausstrahlung entfallenden Antheils, ceteris paribus, der Quadratwurzel aus der Windgeschwindigkeit proportional ist."

Indess erschien diese Schlussfolgerung doch noch einer gewissen Bestiitigung zu bedürfen. Ein nahliegender Einwand gegen die Beweiskraft der von Schuckmann'schen Verrsuche besteht darin, dass von Schuckmann die von dem Thermometer angezeigte Temperaturerniedrigung der Gesammtwärmeabgabe des Abkühlungskörpers direct proportional setzte. Die Aufstellung dieser Relation ist nur unter der Voraussetzung berechtigt, dass die intermittirende Durchmischung völlig ausreichte, um eine während der ganzen Versuchsdauer in allen Theilen gleichmässig erkaltende Masse herzustellen. Ob diese Annahme in der That richtig wäre, bedurfte weiterer Versuche mittels einer ausgiebigeren, womöglich dauernden Rührvorrichtung. Dieselbe war auf folgende Weise (s. Fig. 1) leicht anzubringen:

Ich befestigte mittels eines scharfgeschnittenen Schraubengewindes in dem Verschlussstopfen einer Glaskugel ein möglichst glatt polirtes, verti- 
eales Messinglager, welches einem gleichfalls sorgfiltigst polirten Messingstab zur Führung diente. Derselbe trug an seinem freien Ende ein kleines, aus Aluminium hergestelltes Robinson'sches Schalenkreuz von $30 \mathrm{~mm}$ Armlänge und $37 \mathrm{~mm}$ Schalendurchmesser, an seinem anderen, in's Kugelinnere ragenden Ende eine bohnenförmig gestaltete Aluminium-Schaufel von ca. $5^{\mathrm{cm}}$ Länge und ca. $1^{\mathrm{cm}}$ Breite. Dieses (automatische) Rührwerk war so leicht, dass es schon bei Wind von geringer Geschwindigkeit in lebhafte Drehung gerieth.

Zum Versuch wurde der Hals der Kugel in eine Sehlinge aus Draht gelegt, deren um einander gedrehte Enden in S-förmiger Krümmung weiterliefen und mittels Oesen an dem horizontalen Halter eines Stativs.

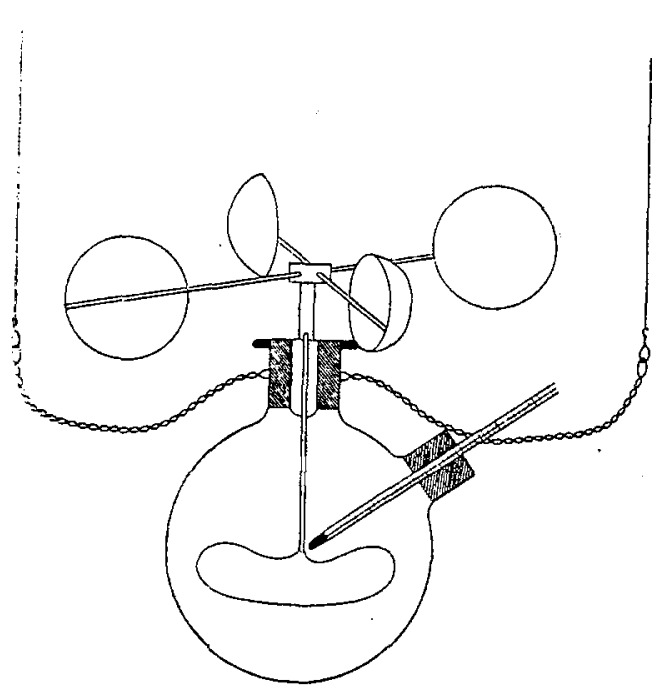

Fig. 1. befestigt wurden, so dass das Abkühlungsobject frei schwebend dem Winde ausgesetzt war.

In allen anderen Versuchsbedingungen habe ich mich an die ron Schuckmann's möglichst enge angelehnt. Meine Glaskugel hatte genau gleichen Durchmesser wie die seinige, ihr Inhalt betrug in versuchsfertigem Zustande $212 \mathrm{grm}$, also nur $3 \mathrm{grm}$ weniger als die von Sebuckmann's; Beobachtungsdauer, Temperaturdifferenz zwischen Abküblungsobject und Zim-

merluft, Versuchsraum, Tretwerk und Ventilator, ja sogar der das Tretwerk versehende Diener waren die gleichen; ebenso waren Temperatur und Feuchtigkeit im Versuchsraum dieselben wie zur Zeit der früheren Versuche.

Tabelle III.

\begin{tabular}{r|c|c|c|c|c}
\hline$v=$ & 23 & 30 & 38 & 51 & \\
\hline$T=15^{\circ}$ & 2.5 & 2.6 & 2.9 & 3.6 & $=4$ \\
25 & 4.1 & $4 \cdot 6$ & 5.4 & 6.1 & $=4$ \\
35 & 6.4 & 6.6 & 7.8 & & $=4$
\end{tabular}

Die Ergebnisse meiner Versuche sind in Tabelle III zusammengestellt. 
Einfluss des Windes auf die Wärmeabgabe todter Objecte. 203

In derselben bedeutet, nach dem Vorgange von Schuckmann's, $T$ die Differenz zwischen Object- und Zimmertemperatur, $\vartheta$ die in Celsiusgraden ausgedrückte Temperaturerniedrigung des Objectes, $v$ die Windgeschwindiglieit in Decimetern.

Vergleicht man von Sehuckmann's und meine Ergebnisse, so fällt auf, dass die letzteren durchwegs höhere Werthe aufweisen. Da mit Ausnahme der Rührvorrichtung alle Versuchsbedingungen durchaus übereinstimmten, so ist zweifellos der Mehrbetrag in meinen Versuchen auf die gründlichere Durchmischung des Kugelinhaltes zurückzuführen, während von Schuckmann's nur zeitweilig in Bewegung gesetztes Rührwerk offenbar keine so grosse Wirkung hatte. Wird durch diese Differenz von Schuckmann's Schlusssatz in Frage gestellt oder nicht? Um dies zu entscheiden, muss zunächst, in Analogie mit von Schuckmann's Berechnungen, der auf die Strahlung entfallende Theil der Wärmeabgabe von der Gesamtwärmeabgabe in Abzug gebracht werden. Diese Reduction ist in Tabelle IV, worin $\vartheta_{L}$ Temperaturerniedrigung durch Leitung bedeutet, geschehen.

Tabelle IV.

\begin{tabular}{|c|c|c|c|c|c|}
\hline$v=$ & 23 & 30 & 38 & 51 & \\
\hline$T=15^{\circ}$ & $2 \cdot 2$ & $2 \cdot 3$ & $2 \cdot 6$ & $3 \cdot 3$ & $=\mathscr{\vartheta}_{\mathrm{L}}$ \\
\hline 25 & $3 \cdot 6$ & $4 \cdot 1$ & $4 \cdot 9$ & $5 \cdot 6$ & $=t L$ \\
\hline 35 & $5 \cdot 6$ & $5 \cdot 8$ & $7 \cdot 0$ & & $=4_{L}$ \\
\hline
\end{tabular}

Sollte vou Schuckmann's Gesetz Gültigkeit auch für diese Werthe haben, so musste für alle Versuche mit gleichem $T$ der Quotient $\frac{\vartheta L}{\sqrt{v}}$ gleich sein. Diese Forderung ergiebt sich aus folgender Ueberlegung: Gesetzt, die Wärmeabgabe im Wind gegen die in Windstille würde proportional der Wurzel aus der Windgeschwindigkeit gesteigert, so wäre, wenn man die Wärmeabgabe in der Windstille $k$ (d. i. Konstante) nennt, z. B. für die Versuche mit $T=15^{\circ}$ folgende Reihe von Gleichungen aufzustellen:

$$
\begin{array}{lll}
2 \cdot 2=k \sqrt{23} & \text { oder } & \frac{2 \cdot 2}{\sqrt{23}}=k \\
2.3=k \sqrt{30} & , & 2 \cdot 3 \\
& & \sqrt{30}=k \\
2 \cdot 6=k \sqrt{38} & \Rightarrow & \frac{2 \cdot 6}{\sqrt{38}}=k \\
3 \cdot 3=k \sqrt{51} & , & \frac{3 \cdot 3}{\sqrt{51}}=k,
\end{array}
$$

d. h. für alle Versuche mit gleichem $T$ müsste $\frac{\vartheta L}{V_{v}}$ konstant sein. 
Führt man die Berechnung dieser Quotienten aus, so ergeben sich die in Tabelle $V$ eingetragenen Werthe.

Tabelle V.

\begin{tabular}{c|c|c|c|c|c}
\hline$v=$ & 23 & 30 & 38 & 51 & \\
\hline$T=15^{\circ}$ & 0.46 & 0.42 & 0.42 & 0.46 & $=\frac{i+\mathrm{L}}{V}$ \\
25 & 0.76 & 0.75 & 0.79 & 0.78 & $=\frac{i \mathrm{~L}}{\sqrt{v}}$ \\
35 & 1.19 & 1.07 & 1.12 & & $=\frac{i \mathrm{~L}}{V}$
\end{tabular}

Demnach zeigt sich zwar bei $T^{\prime}=15$ und $T=25^{\circ}$ eine befriedigende Uebereinstimmung der Quotienten $\frac{\vartheta}{V_{v}}$ und eine annähernde Analogie mit von Schuckmann's Werthen, aber nicht ganz bei $T=35^{\circ}$. Hier treten Abweichungen von 0.05 und 0.06 vom Mittelwerth auf und legen deu Gedanken nahe, dass der Versuchsanordnung, ausser dem oben erhobenen Einwand unzureichender Durchmischung, noch geringfügige weitere Mängel anhaften, die bei höherem $T$ vielleicht deutlicher hervortreten könnten.

Der nächstliegende Einwand betrifft die Kürze der Beobachtungszeit. Es könnte vermuthet werden, dass die Ergebnisse bei grösserer Versuchsdauer andere gewesen wären. Allein diese Verlängerung wurde absichtlich vermieden, um die Reactionstemperatur des Körpers nicht allzu sehr von seiner Anfangstemperatur zu entfernen. Hierin aber liegt der schwerwiegendste, principielle Fehler der Anordnung: $T$ ist keine konstante Grösse, sondern erleidet eine stetige Aenderung. Der Wind trifft demnach im Laufe des Versuches nicht einen gleichen, sondern in jedem Augenblicke bezüglich seiner Wärmeabgabe anders beschaffenen Körper und wird demgemäss auch anders wirken. Wenn auch durch Festsetzung der Beobachtungszeit auf nur 5 Minuten der Fehler möglichst verringert wurde, so war er doch nie auszuschalten und musste bei besserer Durchmischung und steigendem $T$ sogar an Einfluss gewinnen. Hieraus folgt, dass die aus diesen Versuchen abgeleitete Gesetzmässigkeit noch nicht als völlig erwiesen gelten kann. $\mathrm{Da}$ sie jedoch weiteren, bedeutsamen Folgerungen zur Grundlage dienen sollte, so erachtete Herr Geimrath Flügge ihre Nachprüfung unter Ausschaltung der besprochenen Mängel für unerlässlich und gab die Anregung zu einer weiteren Versuchsreibe nach anderem Princip, über die im Folgenden berichtet werden soll. 
EINfluss des Windes a dF de Wärdeabgabe todter ObJeCte. 205

Der leitende Gedanke des neuen Versucbsplanes war, die wärmeentziehende Wirkung des Windes nicht, wie bisher, an dem Temperaturabfall eines abkühlenden Körpers zu messen, sondern vielmehr ein Object ron constant bleibender Temperatur dem Winde auszusetzen und den erhöhten Wärmeverbrauch zn bestimmen, den es im Kampfe mit dem Windanfall zur Erhaltung seiner Wärme benöthigte. Der Ueberschuss von Calorien, welcher sich, unter sonst ganz gleichen Versuchsbedingungen, im Wind gegenüber der Windstille ergab, musste dann genau der Wärmemenge entsprechen, welche dem Körper durch den Wind entzogen worden war. -

Das Versuchsobject bestand aus einem, mit warmem Wasser gefüllten, rertical aufgestellten Cylinder von $147.5^{\mathrm{mm}}$ Durchmesser und $201^{\mathrm{mu}}$ Höhe, welcher aus $1^{\mathrm{mm}}$ starkem Nessingblech hergestellt und aussen glatt polirt war (s. Fig. 2). Seine untere ebene Fläche hatte einen runden Ausschnitt von $39 \mathrm{~mm}$ Durchmesser, über dem sich nach einer geringen Verjüngung ein $3.5 \mathrm{~cm}$ in Durchmesser haltendes, in mehreren zickzackförmigen Windungen nach oben durch den Körper ziehendes Messingrohrerhob, durch die Cylinderdecke hindurchtrat und, noch $35^{\mathrm{cm}}$ frei fortgeführt, wie ein schräger Schornstein mit einer Neigung von ca. $60^{\circ}$ über den Körper emporragte. In der Wand des Schornsteins befand sich dicht oberhalb seines Ursprunges eine Durchbohrung, in welcher ein Thermometer luftdicht angebracht war. Die Schornsteinaussenfläche war mit einer dicken Schicht von Kieselguhrmasse sorgfältig umkleidet. -

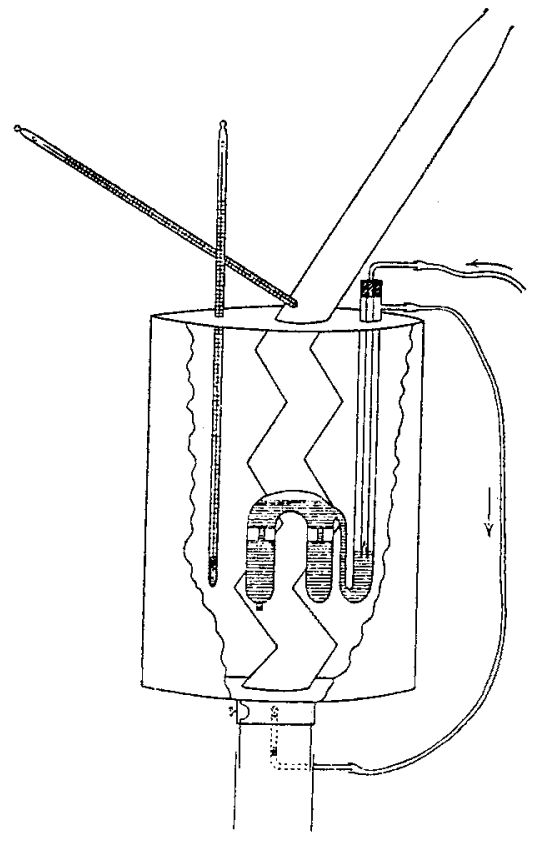

Fig. 2. Ausser der Durchtrittsöffnung der Heizschlange war der Deckel noch mit einer Reihe von Durchbohrungen für Thermometer, Thermoregulatoren, sowie für die Ein- und Ausströmungsrohre der zur Durchmischung des Wassers durchgetriebenen Luft versehen, wovon unten noch ausführlich die Rede sein wird. - Der Cylinder war stets mit der gleichen Wassermasse, $2500^{\mathrm{grm}}$, gefüllt. Er ruhte auf einem weiten Ringe aus schmalem Eisen auf, welcher an einem Stativ mit beschwertem Fuss angebracht 
war. Im Uebrigen hielt sich der Körper völlig frei in seiner Stellung; auf deren Constanz während der ganzen Dauer der Versuche aufmerksam geachtet wurde.

Die Erwärmung des Versuchsobjectes geschah mittels Leuchtgas, welches vorher eine grosse, frisch geprüfte Elster'sche Gasuhr (s. Fig. 3, $G_{1}$ ) passirte.

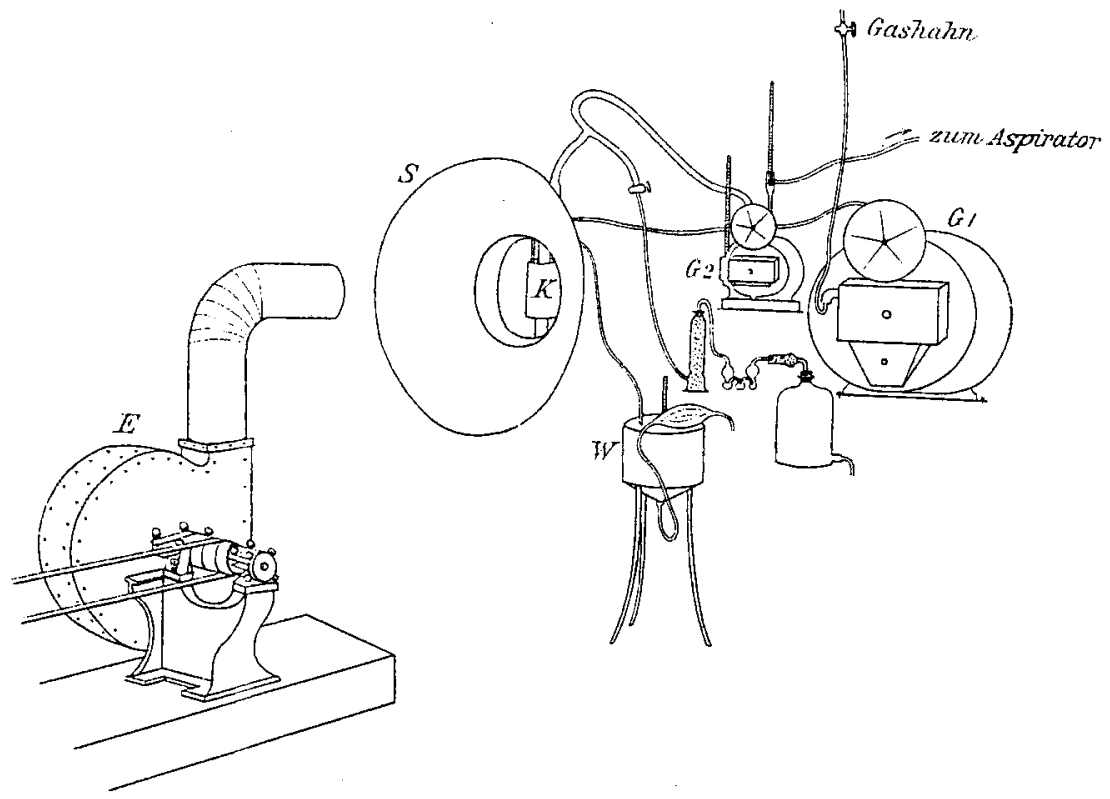

Fig 3

$G_{1}$ Grosse Gasuhr. $G_{2}$ Kleine Gasuhr. $K$ Versuchskörper. $\quad W$ Warm wasserbad. $E$ Exhaustor. $S$ Blechschirm.

Aus der Gasuhr trat das Gas in einen, im Wasser des Versuchskörpers $(K)$ versenkten Aether-Quecksilberregulator eigenartiger Form und Construction (s. Fig. 2), der nach meinen Angaben in zweckentsprechender Weise von Dr. Rohrbeck, Berlin, angefertigt war. Aus dem Regulator strömte das Gas in einen kleinen Bunsenbrenner, welcher in einem, luftdicht an den Versuchskörper anschliessenden und windgeschützt endendem, Rohre geborgen war und mit kleiner, blauer, ruhiger, nie russender Flamme brannte. Die hierbei entwickelten Heizgase zogen durch die Zickzackschlange empor und verliessen den Körper durch den Schornstein.

Die Durchmischung des Wassers im Abkühlungskörper geschah bei den höheren, der Körperwärme des Menschen naheliegenden Temperaturen in sehr einfacher Weise durch Einblasen von Luft mit dem IIunde. Bei 
Einfluss des Windes dUF die Wärneabgabe tod'cer ObJecte. 207

niedrigerer Finstellung des Thermostaten wurde im Warmwasserbade (7) vorgewärmte Luft mit Hülfe eines starken Gummidruckballes durch algefeuchtete Gummischläuche in das Wasser hineingetrieben. Die Durchmischung erfolgte mit peinlicher Regelmässigkeit jede halbe Minute ca. 10 Sec. lang.

Nach Herstellung eines geeigneten Versuchsobjectes und einwandsfreier Vorrichtungen zur Bestimmung der Wärmeproduction erforderte nunmehr die Bestimmung der in den Schornsteingasen ungenutzt abziehenden Wärmemenge, des Wärmeverlustes, noch die Zuziehung weiterer, complicirter Apparate. Wie leicht verständlich, liess sich der calorische Werth der Schornsteingase aus ihrer Masse und ihrer Temperatur berechnen. Während die letztere an dem Schornsteinthermometer ohne Weiteres abzulesen war, war die für die Bestimmung der Luftmenge einfachste, anemometrische, Methode nicht anwendbar; denn einerseits war unter gewissen Versuchsbedingungen der Auftrieb der Heizgase ein so geringer, dass selbst ein feines Recknagel'sches Anemometer noch nicht in Rotation gerieth, andererseits aber wurde, sobald die Flamme und damit auch die Geschwindigkeit der Heizgase wuchs, die Hitze der letzteren eine so hohe, dass die Einführung ron Prācisionsanemometern auf längere Zeit ausgeschlossen war. Versuche mit Recknagel's Differential-Manometer führten gleichfalls zu keinem befriedigenden Ergebniss. Wollte man an dem Princip der Wärmeberechnung aus Luftmenge und Temperatur überhaupt festhalten und nicht etwa die calorimetrische oder anthracometrische Methode einschlagen, so blieb daher nichts übrig, als die Schornsteingase durch eine 2. Gasuhr zu leiten. Allerdings machte sich auch hier der Uebelstand geltend, dass der Druck der Heizgase zur Drehung der Gasuhr nicht ausreichte; doch war diese Schwierigkeit dadurch zu beseitigen, dass die Abströmungsöffnung der Gasuhr noch mit einer Aspirationsvorrichtung verbunden wurde, mit deren Hülfe nunmehr der Widerstand der Gasulhr überwunden wurde.

Die Anordnung gestaltete sich demnach folgendermaassen: Aus dem Schornstein traten die Gase durch einen dickwandigen Gummischlauch in eine kleine Elster'sche Gasubr $\left(G_{2}\right)$ von 6.5 Liter Capacität, welche mit Thermometern zur Beobachtung der Temperatur des Wassers und der austretenden Gase versehen war. Dieselben wurden sodann nach einem, an die Wasserleitung angeschlossenen Trommelwassergebläse angesogen, aus dem sie schliesslich, zusammen mit dem durchströmenden Wasser, austraten. Die von dem Aspirator geförderte Luftmenge war ausreichend regulirbar; sie betrug, je nach den Versuchsbedingungen, 8 bis 12 Liter pro Minute. 
Zur Erregung des Windes waren die für die Kugelversuche benutzten einfachen Vorrichtungen nicht anwendbar. Die Grösse des Abkühlungskörpers erforderte einen grösseren Querschnitt des Luftstromes, dieser wiederum einen stärkeren Motor, wenn man gleichzeitig auch höhere Geschwindigkeiten erzielen wollte. Gurade bei diesen aber erwies sich die Anwendung eines Schraubenrentilators als grober Fehler. Denn die, schon früher etwas störend empfundene, Centrifugalwirkung seiner zur Axe schräg gestellten Flügel äusserte sich dann derart, dass in der Verlängerung der Axe geradezu eine windstille Zone, ja eine Umkehr der Luftbewegung auftrat, während von da bis zur Peripherie die Bewegung von sehr geringen bis ausserordentlich grossen Geschwindigkeiten schnell anstieg. Nach zahlreichen zeitraubenden Vorversuchen erwies sich schliesslich folgende Anordnung brauchbar, bei deren Einrichtung mich die Herren Abtheilungsvorsteher der Firma Gebr. Körting in dankenswerther Weise mit ihrem fachmännischen Rath unterstützten.

Die Erzeugung des Windes geschah durch einen tangential wirkenden Exhaustor folgender Construction (s. Fig. 3,E). In einem $55^{\mathrm{cm}}$ hohen, gusseisernen Gehäuse bewegte sich ein mühlradartiges Flügelrad von $40^{\mathrm{cm}}$ Durchmesser; die Schaufein hatten eine Breite von $46^{\mathrm{mm}}$, eine Länge von $102 \mathrm{~mm}$. In dem Centrum der einen Gehäusefläche befand sich eine runde Oeffnung von'147 mm Durchmésser (die „Saugeöffnung“"), in der Peripherie des Gehäuses eine gleich weite, nach oben gerichtete (die „Ausblaseöffnung"). Letztere war mit einem kurzen Rohransatz versehen; auf denselben wurde ein knieförmig gebogenes Blechrohr ron $165 \mathrm{~mm}$ lichter Weite und je $44^{\mathrm{cm}}$ Schenkellänge aufgesteckt, dessen freie Oeffnung einem, in einer Entfernung von $1^{\mathrm{m}}$ vertical aufgestellten, grossen Blechschirm $(S)$ genau centrirt gegenüberlag. Derselbe stellte den Mantel eines abgestumpften Kegels von $25^{\mathrm{cm}}$ Höhe dar, dessen grössere, dem Exhaustor zugewandte Grundfläche einen Durchmesser von $100^{\mathrm{cm}}$ hatte, während der Durchmesser der kleineren nur $40^{\mathrm{cm}}$ betrug. Dieser Schirm hatte die Aufgabe, die peripheren, langsameren Strahlen des ron der tusblaseöffnung ausgehenden Kegels bewegter Luft in einem engeren Querschnitt zu sammeln und mit dem schnelleren Axenstrom zu vereinigen. Derselbe trat durch die kleinere Grundfläche des Kegels in ein $15 \mathrm{~cm}$ langes, horizontales Ansatzrohr, an dessen Ausgang der Abkühlungskörper aufgestellt war und hier auf seiner gesammten, dem Exhaustor zugewandten Oberfläche von Luftströmen nahezu gleicher Geschwindigkeit getroffen wurde.

Der Antrieb des Exhaustors geschah durch einen Elektromotor von 1 P.S., welcher mit der Flügelradase durch Riementransmission indirect verkuppelt war. Durch geeignete Vorschaltwiderstände (Glühlampen ver- 
EINFLUSS Des WINDES aUf DIE WäRMeabGabe tOdTER OBJECTE. 209

schiedener Kerzenstärke) konnte seine Tourenzahl nach Belieben regulirt und die Geschwindigkeit der den Versuchskörper treffenden Luftströme durch alle Stufen von $0.80^{\mathrm{m}}$ bis $9^{\mathrm{m}}$ pro Secunde gewechselt werden. Für noch langsamere Luftströme wurde statt des oben beschriebenen Ansatzrohres von gleichbleibendem Querschnitt ein trompetenartig sich erweiterndes Rohr aufgesetzt, welches eine Verlangsamung der Luftströme bis zu 0.40 bis $0.50 \mathrm{~m}$ pro Secunde ermöglichte.

Vergegenwärtigt man sich nun nochmals, was dieser complicirte Apparat zu leisten hatte, so ergeben sich folgende Anforderungen:

Es musste möglich sein,

1. langdanernde Versuche in Windstille und Wind an einem Körper von constant bleibender, in allen Theilen gleichmässiger Temperatur auszuführen;

2. die jeweils zugefübrte Gasmenge zu bestimmen und ihren calorischen Werth unter den jeweiligen Versuchsbedingungen zu berechnen;

3. die Menge und Temperatur der gleichzeitig abziehenden Schornsteingase zu bestimmen und den durch sie bedingten Calorienverlust zu berechnen;

4. Luftströme von gleichbleibender und im Querschnitt gleichstarker Geschwindigkeit auf das Versuchsobject wirken zu lassen und die Geschwindigkeit von Versuch zu Versuch nach Belieben zu wechseln. -

Wie weit meine Versuchsanordnung diesen Aufgaben gereeht wurde, ergiebt sich aus den beigefügten Protokollen, zu deren besserem Verständniss ich die kurze Beschreibnng von der Ausführung eines einzelnen Versuches vorausschicke:

Zunächst wurde der Thermostat auf eine gewisse Temperatur eingestellt. Hielt sich dieselbe constant, so wurde minutenweise, vorerst ohne Wind, folgende Reihe von Ablesungen in regelmässiger Folge ausgeführt: der Stand des Thermostatthermometers, des Schornsteinthermometers, der kleinen Gasuhr, der grossen Gasuhr. Nach Kurzem war ich soweit geübt, dass diese Ablesungen innerhalb von 15 Secunden, im 1. Viertel jeder Minute, erledigt waren. Im 2. Viertel wurde eine gründliche Durchmischung des Thermostatwassers vorgenommen; die nun folgende Pause des 3. Minutenviertels konnte zur Feststellung der Temperatur and Feuchtigkeit des Versuchsraumes mittels Assmann's Aspirationsthermometer und Koppe'schen Haarhygrometers benutzt werden, während im 4. Viertel nochmals gründlich durchgemischt wurde.

Waren diese Beobachtungen ohne Störung, zumeist 1 Stunde lang, fortgeführt, so wurde nunmehr der Motor in Bewegung gesetzt, seine Geschwindigkeit auf eine gewisse Stärke regulirt und, nach dem zunächst 
Tabelle VI.

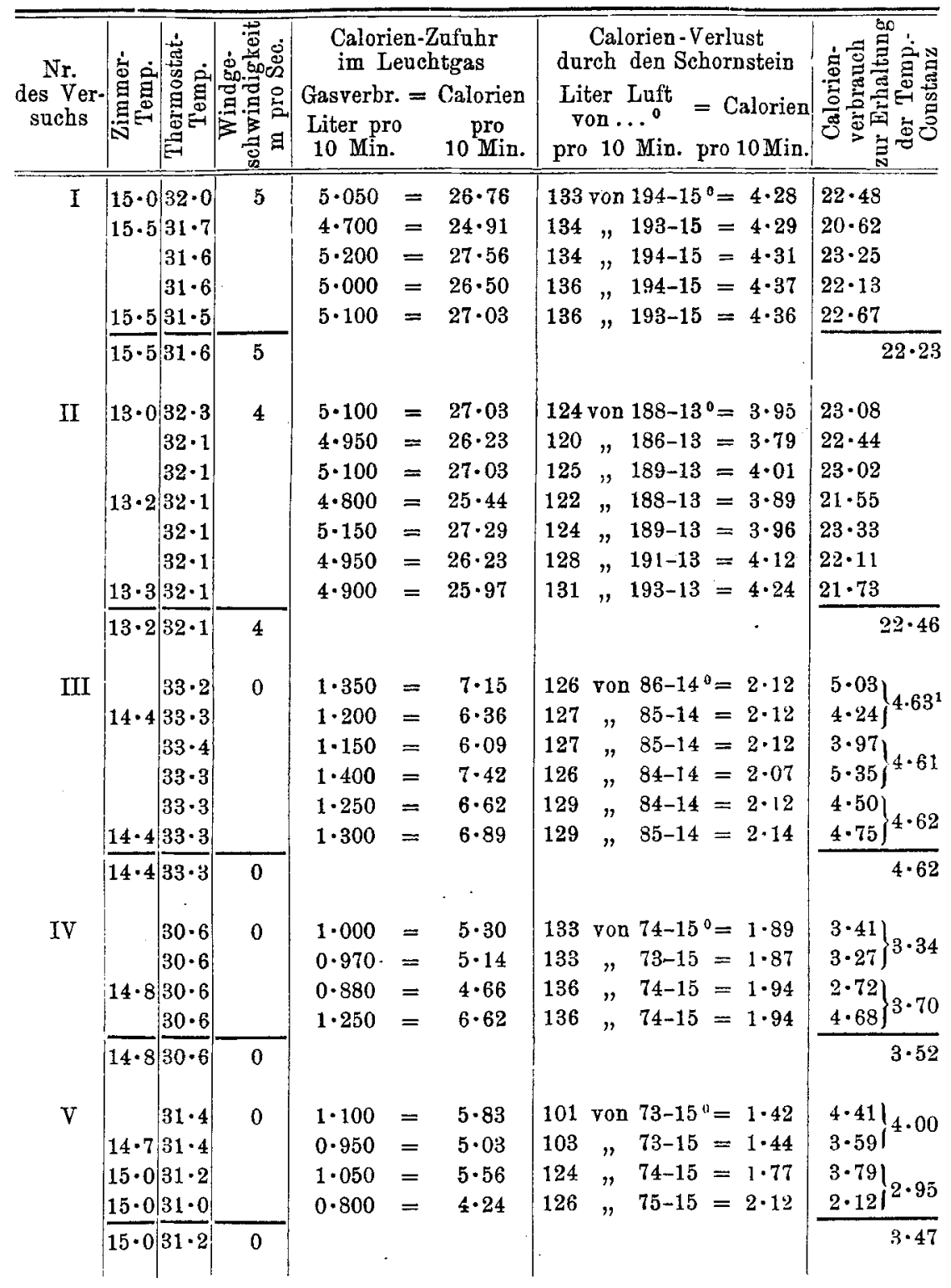

1 In den Versuchen mit geringem Gasverbrauch sind mehrfach zur Bildung des Mittels $2 \times 10$ Minuten zusammengezogen, weil die innerhalb von 10 Minuten verbrauchte geringe Gasmenge eine gleichmässige Drehung der Gasuhr nicht bewirkte und erst in den nächsten 10 Minuten der Gesammtrerbrauch beider Versuchsperioden 
Ennfluss des Windes auf die Wärnueabgabe todter ObJecte. 211

Tabelle VI. (Fortsetzung.)

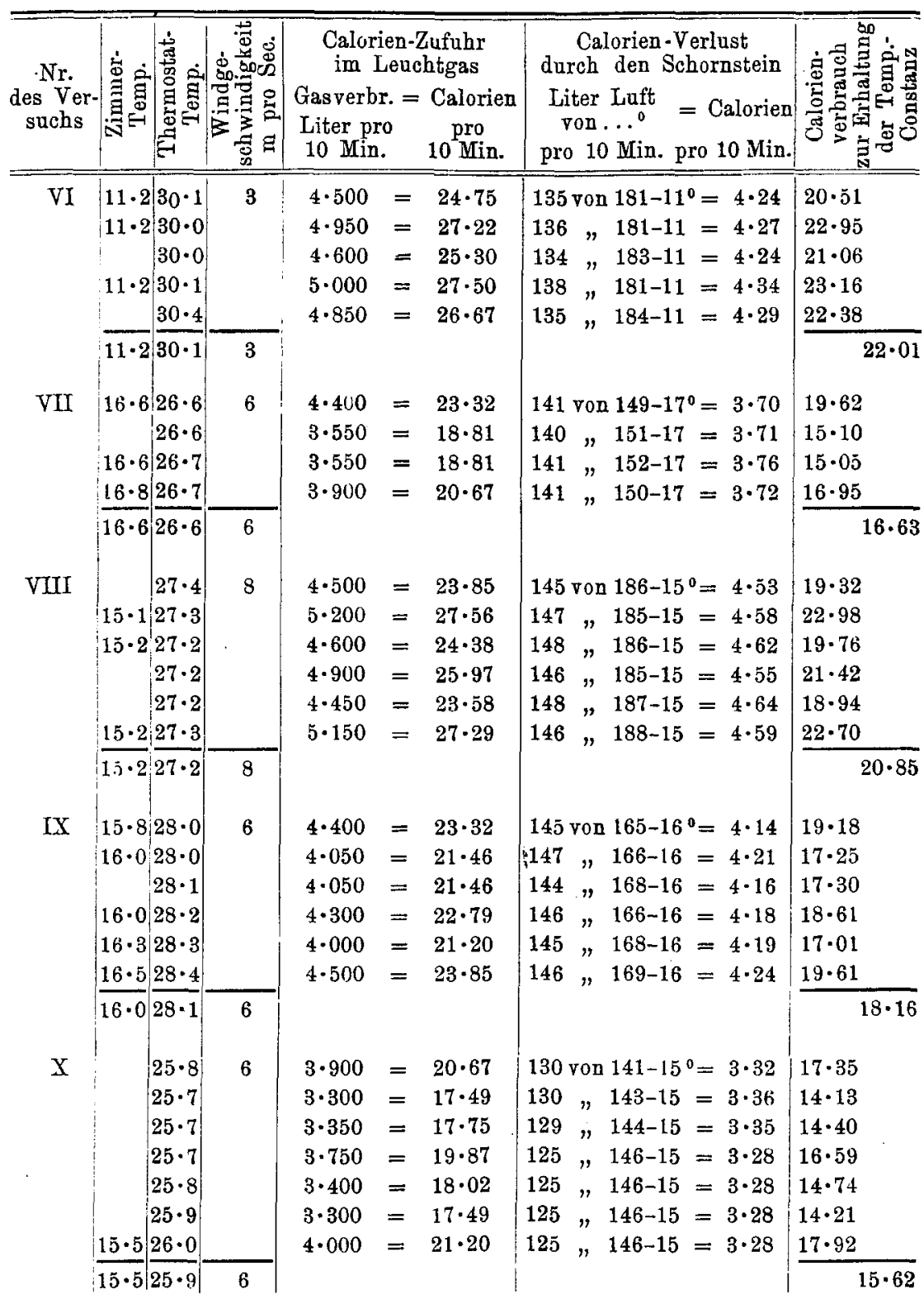

zum maassgebenden Ausschlag führte. Dass gleichwohl in den einzelnen Versuchsperioden die Verbrennung eine gleichmässige war, geht aus den wenig schwankenden Werthen für den Calorienverlust hervor. 
Tabelle VI. (Fortsetzung.)

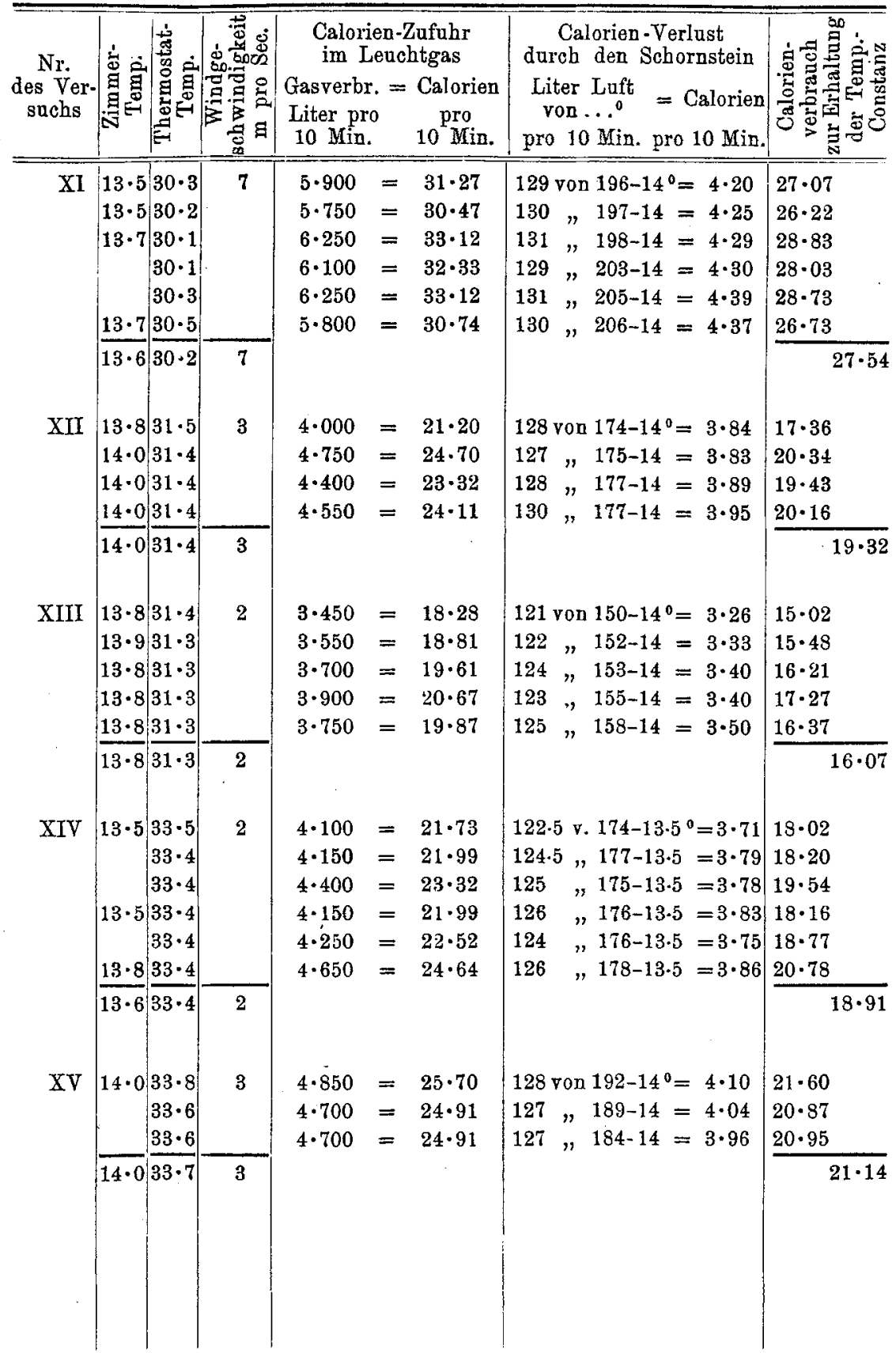


Einfluss des Windes auf die Wärmeabgabe todter Objecte. 213

Tabelle VI. (Fortsetzung.)

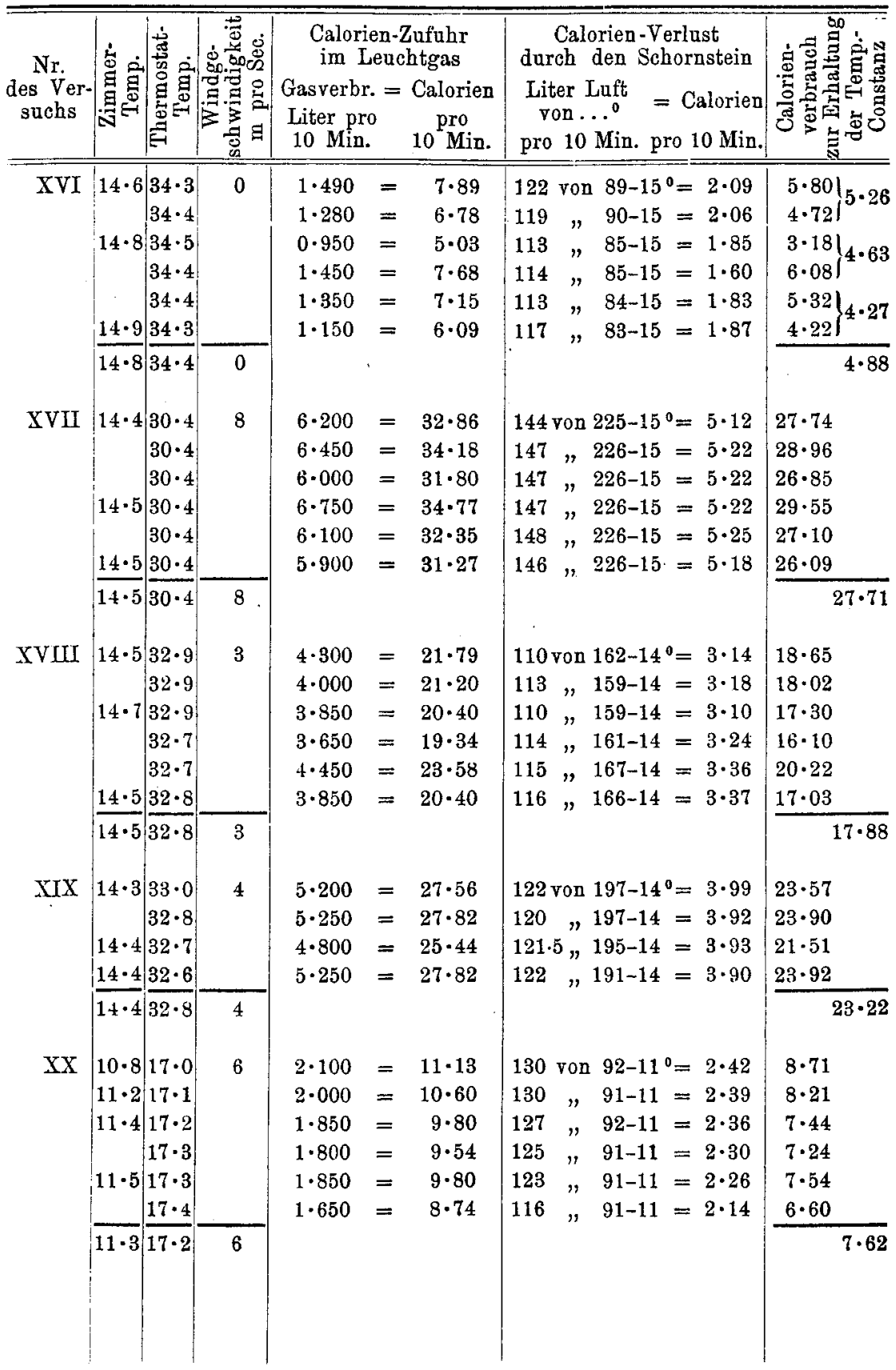


T abelle VI. (Fortsetzung.)

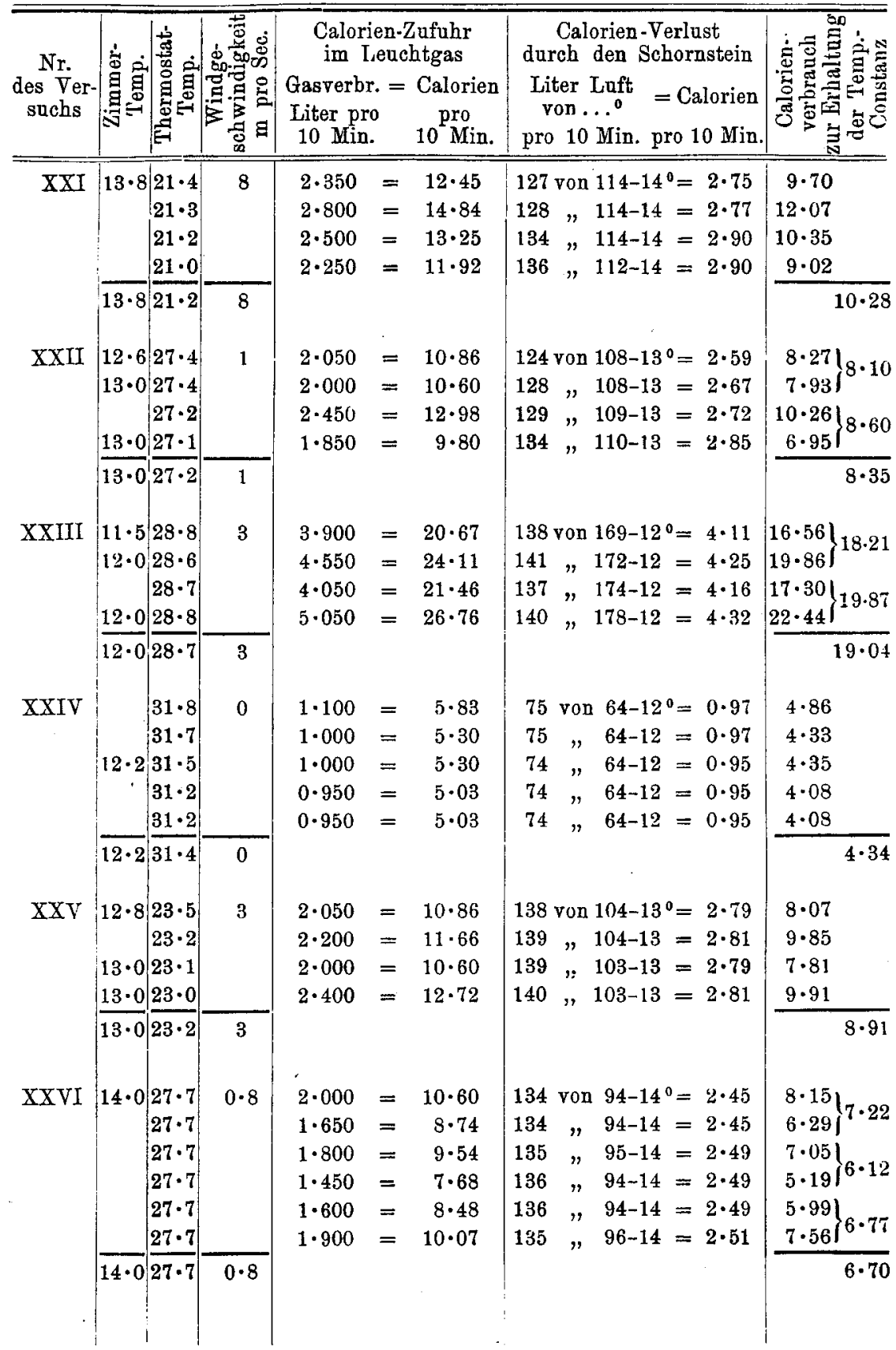


Einfluss des Windes aUf die Wärmeabgabe todter Objecte. 215

Tabelle VI. (Fortsetzung.)

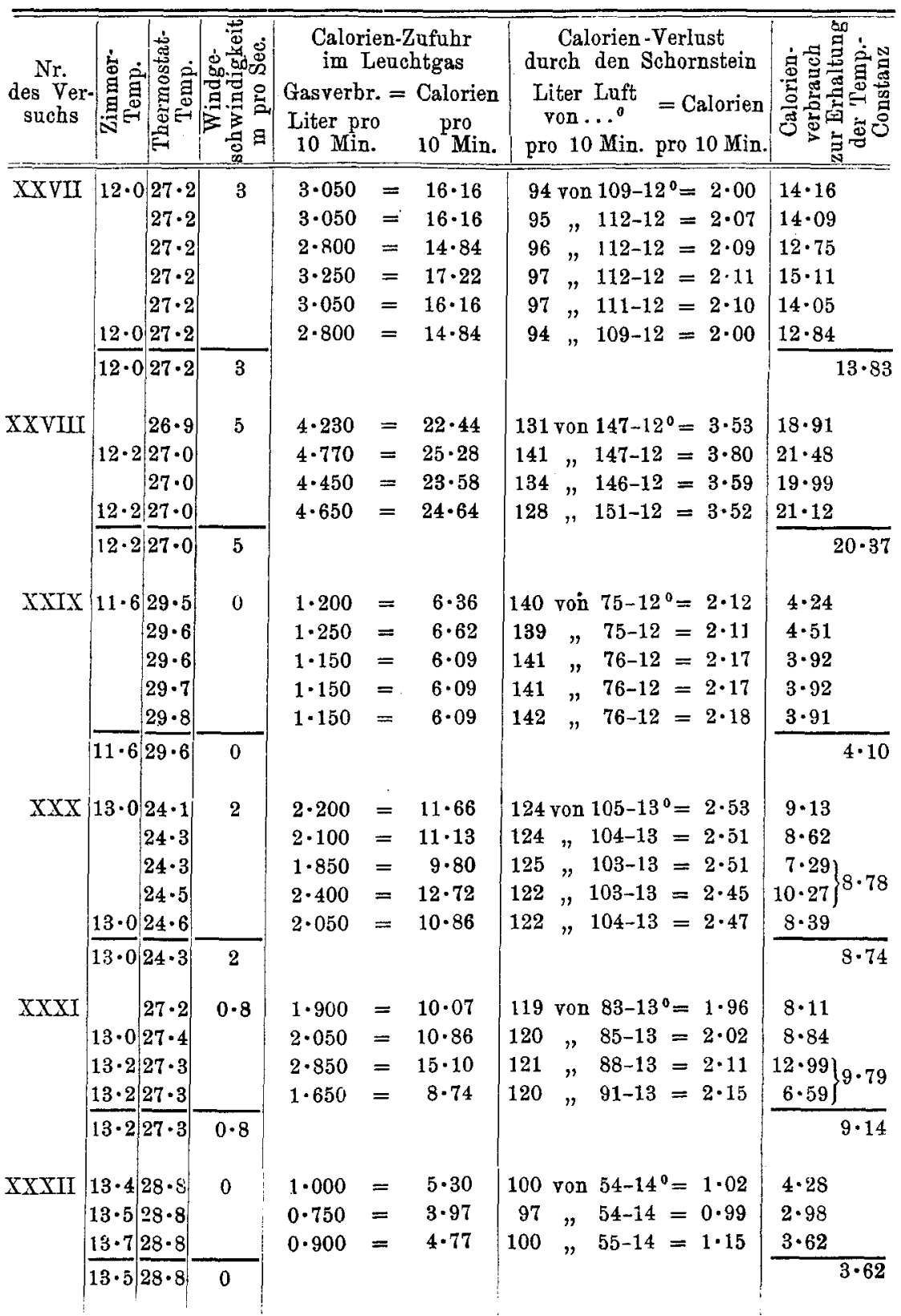


Tabelle VI. (Schluss.)

\begin{tabular}{|c|c|c|c|c|c|c|c|}
\hline $\begin{array}{l}\text { Nr. } \\
\text { des Ver } \\
\text { suchs }\end{array}$ & - & 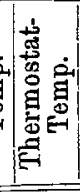 & 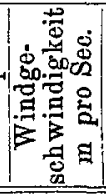 & $\begin{array}{l}\text { Calorier } \\
\text { im Le } \\
\text { Gasverbr. } \\
\text { Liter pro } \\
10 \text { Min. }\end{array}$ & $\begin{array}{l}\text { en-Zufuhr } \\
\text { euchtgas } \\
=\text { Calorien } \\
0 \quad \text { pro } \\
10 \text { Min. }\end{array}$ & $\begin{array}{l}\text { Calorien-Verlust } \\
\text { durch den Schornstein } \\
\text { Iiter Luft = Calorien } \\
\text { von } \ldots 0^{\circ}=\text { Min. pro } 10 \text { Min. } \\
\text { pro } 10 \text { Min. }\end{array}$ & 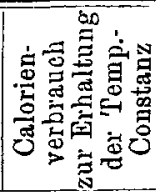 \\
\hline \multirow[t]{2}{*}{ XXXПI } & 14.4 & $\left|\begin{array}{l}26 \cdot 5 \\
26 \cdot 5 \\
26 \cdot 4 \\
26 \cdot 4\end{array}\right|$ & 0.6 & \multirow[t]{2}{*}{$\begin{array}{l}2 \cdot 200 \\
1 \cdot 000 \\
1 \cdot 850 \\
1 \cdot 150\end{array}$} & \multirow[t]{2}{*}{$\begin{array}{l}=11 \cdot 66 \\
=\quad 5 \cdot 30 \\
=\quad 9 \cdot 80 \\
=\quad 6.09\end{array}$} & \multirow[t]{2}{*}{$\begin{array}{l}110 \text { von } 74-14^{0}=1 \cdot 59 \\
109 \Rightarrow 72-14=1 \cdot 53 \\
110 " 72-14=1 \cdot 54 \\
110 \Rightarrow 73-14=1 \cdot 57\end{array}$} & $\begin{array}{l}10 \cdot 07\} 6 \cdot 92 \\
3 \cdot 77 j^{6 \cdot 9} \\
8 \cdot 26\} \\
4 \cdot 52\}^{6 \cdot 39}\end{array}$ \\
\hline & $14 \cdot 4$ & $426 \cdot 4$ & $0 \cdot 6$ & & & & 6.65 \\
\hline
\end{tabular}

erfolgenden Temperaturabfall des Thermostaten, seine Wiedereinstellung auf eine constante, der früheren meist naheliegende Temperatur abgewartet. War dieselbe erfolgt, so wurde wiederum in der oben beschriebenen Weise die Beobachtung mindestens 1 Stunde lang ausgeführt.

In den beigegebenen. Fersuchsprotokollen sind nun die Beobachtungen von 10 Minuten immer zu einem Werthe zusammengefasst, derart, dass Columne 1 die Zimmertemperatur während der Beobachtungszeit angiebt, Columne 2 das Mittel sämmtlicher in 10 Minuten beobachteter Thermostattemperaturen, Columne 3 die Windbewegung in Metern pro Secunde, Columne 4 die in 10 Minuten zugeführte Gasmenge, bezw. die ihr entsprechende Calorienzahl, für deren Berechnung auf Grund der täglichen Bestimmungen durch das städtische chemische Untersuchungsamt ein Durchschnittswerth von 5300 Calorien pro Cubikmeter zu Grunde gelegt wurde. Columne 5 enthält die Menge und Temperatur der in 10 Minuten abziehenden Schornsteingase, sowie die hieraus berechneten, in Verlust gerathenen Calorien. Columne 6 giebt schliesslich die Differenz der unter 4 und 5 resultirenden Calorien an, d. h. die von dem Körper zur Erhaltung seiner Temperatur benöthigte Wärmemenge.

Kehren wir nunmehr zu der oben beregten Frage über die Leistungsfähigkeit der Versuchsanordnung zurück, so ergiebt sich aus den Protokollen:

1. Die geforderte Constanz und gleichmässige Vertheilung der Temperatur des Versuchskörpers ist in befriedigender Weise erreicht worden. Der Thermostat hielt sich oft während der ganzen Dauer eines Versuches auf völlig gleicher Wärme; in den anderen Versuchen betragen die Temperaturschwankungen nur wenige Zehntel eines Grades. Beobachtungen, welche unter einander um mehr als 5 Zehntel eines Grades abwichen, 
wurden nicht verwerthet. Dass auch die Durchmischung des Wassers eine ausreichende war, liess sich aus der Temperaturconstanz schliessen, welche das Thermometer auch in den Durchmischungspausen, also im 1. und 3. Viertel jeder Beobachtungsminute anzeigte. Uebrigens trat auch hier wieder die grosse Bedeutung der ausgiebigen Durchmischung auf's Dentlichste hervor; wurde gelegentlich, in Folge einer Störung, die Durchmischung auch nur 1 Minute lang unterbrochen, so konnte das bis dahin tadellos constant erhaltene Thermometer um $1 / 2$ bis 1 Grad steigen und kehrte erst nach gründlicher Durchmischung wieder allmählich in seine constante Lage zurück.

2. Die zugeführte Gasmenge war an der Gasuhr ohne Weiteres abzulesen. Fraglich war nur, ob es statthaft sei, für die Berechnung der dem Körper zugeführten Calorien den mit dem Calorimeter bei vollständiger Verbrennung festgestellten Wärmewerth in die Rechnung ein. zusetzen, oder ob nicht vielmehr ein Theil des Leuchtgases unverbrannt in den Schornsteingasen entwich. Bei einigen Versuchen wurden daher noch gewisse Mengen Schornsteinluft vermittelst einer Nebenleitung durch Kaliapparate hindurchgeführt und die absorbirte Kohlensäure gewichtsanalytisch bestimmt. Es ergab sich, dass die Verbrennung auch unter sehr verschiedenen Versuchsbedingungen eine gleich intensive und nabezu rollständige war. Dieses günstige Resultat war vermuthlich auf die überaus reichliche Luftzufuhr zu dem Bunsenbrenner zurückzuführen, welche mit Hülfe der Aspiration bewirkt werden konnte.

3. Die Menge und Temperatur der Schornsteingase ergaben sich aus den Thermometer- und Gasometerablesungen. Die Berechnnng der in Verlust gerathenden Calorien (W) geschah aus diesen Daten mit Hülfe der Formel:

$$
W=C P t
$$

worin $C$ die specifische Wärme der Luft $=0 \cdot 238$;

$P$ das Gewicht der Luft in Kilogramm bei der beobachteten Temperatur;

$t$ die Temperaturdifferenz zwischen den Schornsteingasen und dem Zimmer bedeutet; der für $t$ in Frage kommende Subtrahend ist daher jedes Mal zu der Temperatur der Schornsteingase mit beigefügt.

Wie ersichtlich, tritt in diese Berechnung auch die Zimmertemperatur als Factor ein, so dass eine möglichst weitgehende Constanz derselben für die ganze Versuchsreihe wünschenswerth war. Dieses Desiderat war in dem saalartigen, von drei freien Mauern umschlossenen, im Dachgeschoss belegenen Versuchsraum nicht völlig $\mathrm{zu}$ erfüllen. .Immerhin konnten weitaus die meisten Versuche zwischen 13 und $15^{\circ} \mathrm{C}$. durchgeführt 
werden; von den übrigen sind einige bei 11 und $12^{\circ}$, einige bei $16^{\circ}$, ein einziger bei $17^{\circ}$ angestellt, so dass selbst die Extreme nur um $3^{\circ}$ vom Mittelwerth abweichen. Zudem ist die Constanz der Aussentemperatur durchaus kein dringendes Erforderniss, weil in dem Product $C P t$ eine Ungenauigkeit von 0.5 bis 1 für $t$ wenig bedeutet und zweitens für die späteren Berechnungen nicht die absolute Lufttemperatur, sondern die Temperaturdifferenz zwischen Versuchsobject und Versuchsraum in Frage kommt.

4. Die Gleichmässigkeit der Luftbewegung während eines Versuches ist durch die Constanz der Trieblraft gewährleistet. Hingegen gelang es nicht, für alle Windstärken auch Gleichmässigkeit im ganzen Querschnitt des bewegten Luftstromes zu erzielen; trotz der complicirten, zweifellos auch zweckmässigen Vorrichtungen herrschten in dem für das Abkühlungsobject in Betracht kommenden Bereich bei den grösseren Windgeschwindigkeiten noch Unterschiede von ca. $1 / 2 \mathrm{~m}$. Gleichwohl konnte man hoffen, durch recht zahlreiche $\nabla$ ersuche unter möglichst exacten Bedingungen zu brauchbaren Mittelwerthen zu gelangen. -

Zur Erleichterung der weiteren Berechnungen und Folgerungen habe ich aus den Protokollen nochmals lediglich die Endresultate herausgezogen und in Tabelle VII zusammengestellt. Dieselbe enthält in der obersten Horizontalcolumne die Benennung der Windgeschwindigkeit $(v)$, in der ersten Verticalcolumne die für die Wärmeabgabe des Objectes wesentlich interessirende Temperaturdifferenz $(T)$ zwischen Object und Versuchsraum, während die Zahlen im Schnittpunkte einer Horizontal- und Verticalcolumne die unter den einschlägigen Bedingungen von dem Versuchskörper aufgewendeten Calorien angeben, wie sie im Versuche gefunden wurden. Die Tabelle ermöglicht also einen directen Vergleich der in Windstille und Wind unter sonst ganz gleichen Verhältnissen abgegebenen Wärmemengen. Die in der Windstille abgegebene Wärmemenge hätte eigentlich auf theoretischem Wege mit Hülfe der Péclet'schen Tabellen für die constanten Strahlungs- und Leitungscoëfficienten bestimmt werden können. Wie zu erwarten, betrug aber die auf diesem Wege berechnete Calorienmenge mehr als die im Versuch direct beobachtete; beziehen sich doch Péclet's Constantenformeln auf mathematisch geschlossene Idealkörper, während mein Versuchsobject noch zahlreiche Durchbohrungen, Unebenheiten und Trübungen seiner Oberfläche aufwies. Ueberdies äussert sich Ferrini ${ }^{1}$ bezüglich der von Péclet aufgestellten, für uns besonders wichtigen Leitungscoüficienten so kritisch, dass ich es vorzog, von einer theoretischen Berechnung der Wärmeabgabe mit Hülfe von Péclet's

1 Ferrini, Technologie der Wärme. Jena 1878. 
Einfluss des Windes adf die Wärmeabgabe todter Objecte. 219

Tabelle VII.

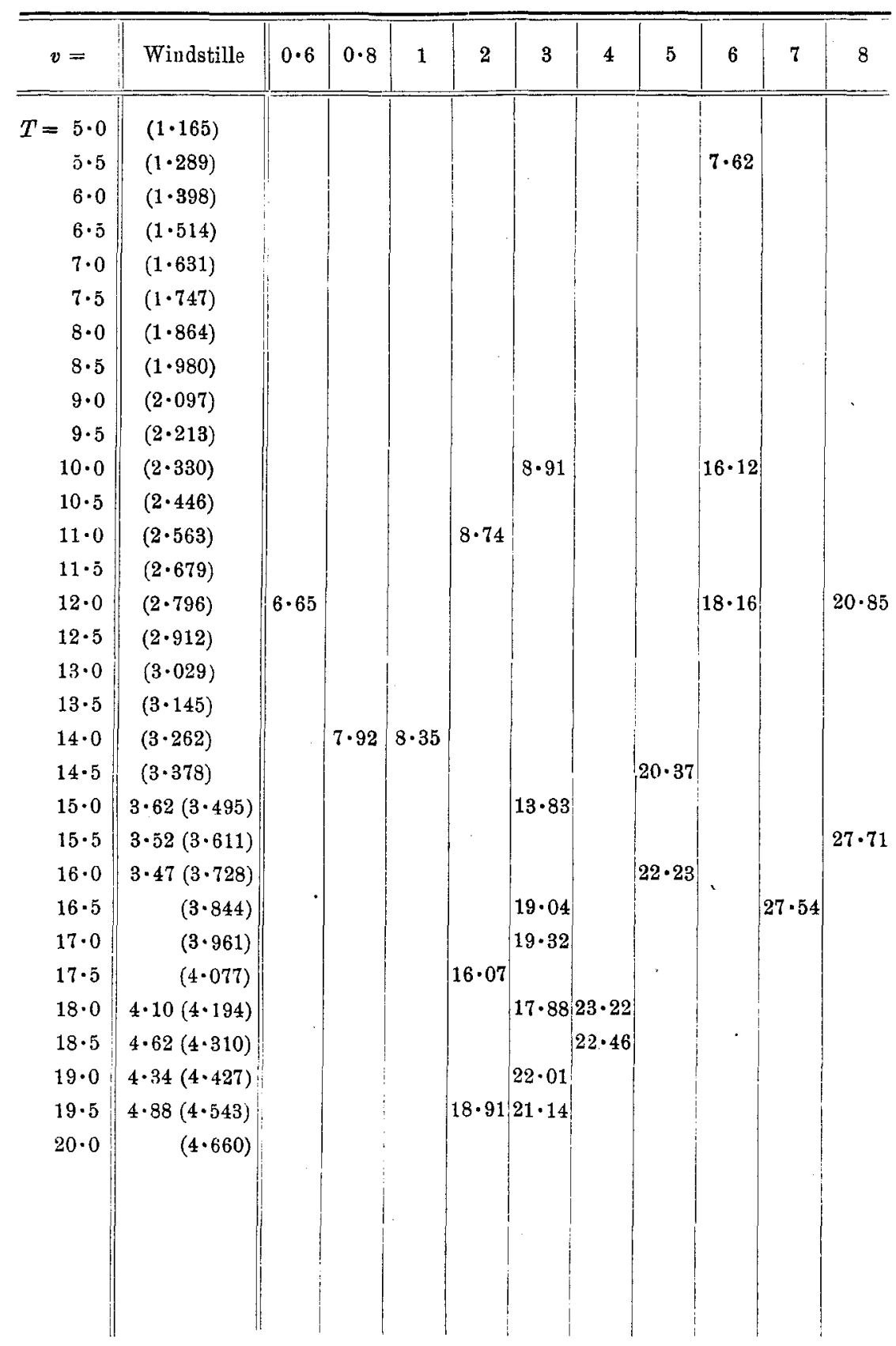


Constanten ganz Abstand zu nehmen und mich lediglich an die im Versuch gewonnenen Zahlen zu halten. Aus diesen war dann unter Benutzung von Péclet's Annäherungsformel: $W=(i+k) \tau 1$, - wo $W$ die abgegebene Wärme in Calorien in der Zeit $\tau, i$ den Strahlungscoëfficienten, $k$ den Leitungscoëfficienten, $T$ die Temperaturdifferenz zwischen Körper und Umgebung bedeutet -, $i+k$ leicht zu berechnen und der Mittelwerth aus allen im Versuch gefundenen Summen $i+k$ zur Aufstellung einer Tabelle (VII) verwerthbar, welche für alle in Betracht kommenden Temperaturdifferenzen $T$ die Wärmeabgabe- des Versuchskörpers in der Windstille anzeigt und zwar die berechneten in Parenthese.

Betrachtet man nun die im Wind gefundenen Calorienzahlen, so fällt zunächst der Anstieg in die Augen, den jede Verticalcolumne proportional dem wachsenden $T$ aufweist, wie dies nicht anders zu erwarten war. Weit interessanter sind die Ergebnisse der Horizontalcolumnen. Auch hier zeigt sich ein Anstieg der Wärmeabgabe, der aber nicht ohne Weiteres der Windgeschwindigkeit proportional gesetzt werden kann, sondern zu derselben in einem anderen, mit wachsender Windstärke zwar auch ansteigendem, aber an Zuwachs relativ stetig abnehmendem Verhältnis steht. So bewirkt z. B. unter sonst sehr ähnlichen Bedingungen ein Wind ron:

$1^{\mathrm{m}}$ pro Sec. eine Wärmeabgabe von $8 \cdot 35$ Calorien pro $10 \mathrm{Min}$.

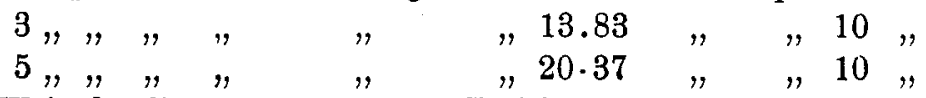

Weit deutlicher mussten diese Beziehungen noch hervortreten, wenn man von den absoluten Werthen absah und sämmtliche Zahlen einer Horizontalcolumne auf die in der Windstille abgegebene Calorienmenge als Einheit bezog, d. h. die Quotienten Wärmeabgabe im Wind $\left(\boldsymbol{W}_{w}\right)$ : Wärmeabgabe in der Windstille $\left(W_{s t}\right)$ berechnete. Diese Berechnung ist in der Tabelle VIII ausgeführt. Dieselbe eirgiebt, dass die Werthe einer Verticalcolumne einander ausserordentlich nahe liegen, ja vereinzelt übereinstimmen, dass also die relative Erhöhung der Wärmeabgabe bei wachsendem Winde, wie sie sich in den Horizontalcolumnen ausspricht, stets die gleiche ist. Es war demnach wohl gestattet, aus sämmtlichen Werthen jeder Verticalcolumne den Mittelwerth zu ziehen und mit dieser neuen Horizontalcolumne die Betrachtungen weiter fortzuführen. Dieselben hatten zum Ziele, die gefundenen Werthe in eine gesetzmässige Relation zur Geschwindigkeit des Windes zu bringen. Im Hinblick auf die an den Glaskugeln von von Schuckmann und mir festgestellte Gesetzmässigkeit war das Nächstliegende, zu versuchen, ob auch die neugewonnenen Ergebnisse der Quadratwurzel aus der Windgeschwindigkeit proportional zu setzen seien. War dies der Fall, so musste, nach der schon früher aus- 
Einfluss des Windes aUf dIE Wärmeabgabe todter ObJecte. 221

Tabelle VIII.

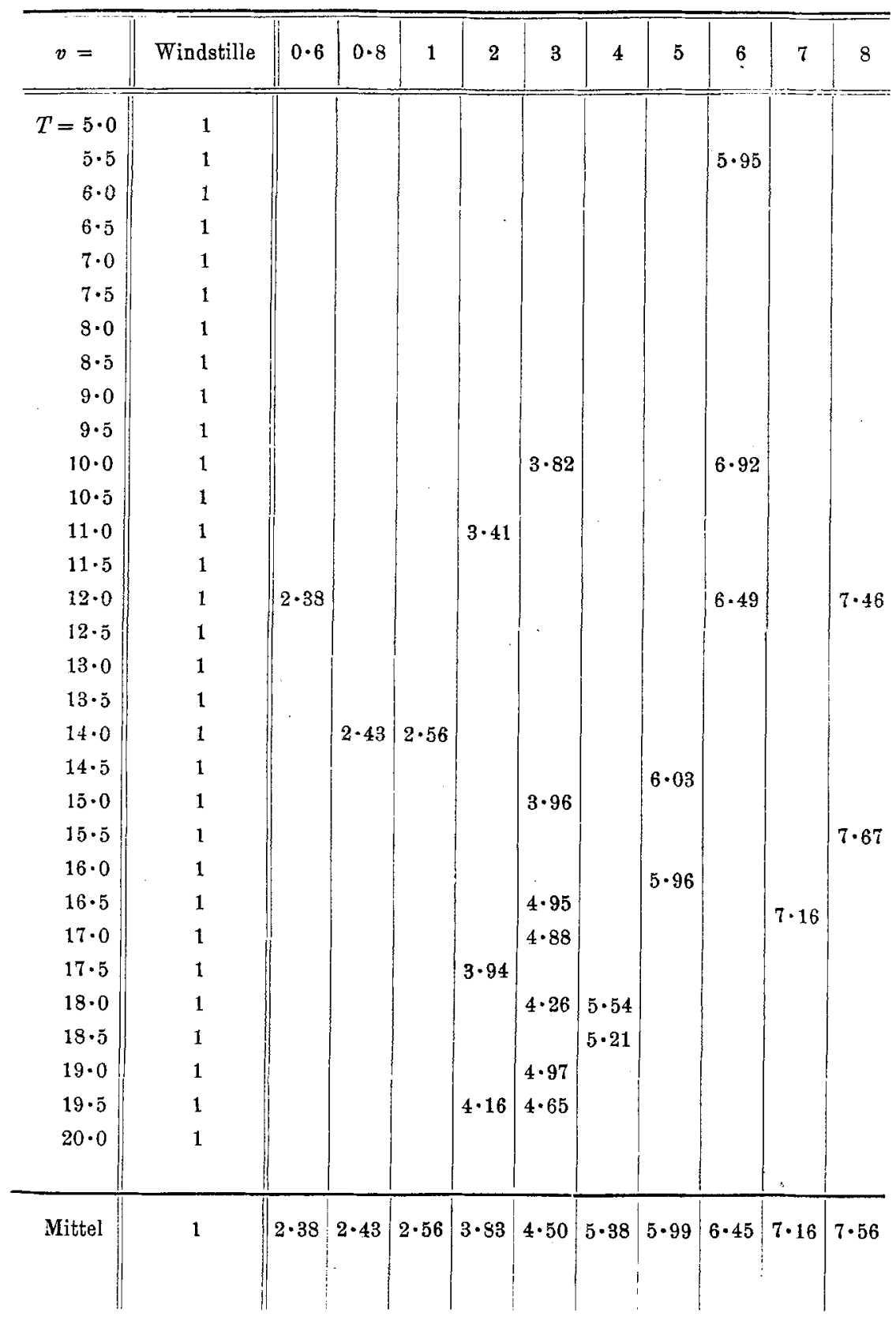


geführten Deduction, der Quotient $\frac{\text { Calorienmenge im Wind }}{\sqrt{v}}$ für alle Wind. stärken constant sein.

Führt man diese Berechnung aus, so ergiebt sich:

$$
\begin{aligned}
& \frac{2 \cdot 38}{\sqrt{0 \cdot 6}}=\frac{2 \cdot 38}{0 \cdot 8}=2 \cdot 97 \\
& \frac{2 \cdot 43}{\sqrt{0 \cdot 8}}=\frac{2 \cdot 43}{0 \cdot 9}=2 \cdot 70 \\
& \frac{2 \cdot 56}{\sqrt{1}}=\frac{2 \cdot 56}{1}=2 \cdot 56 \\
& \frac{3 \cdot 83}{\sqrt{2}}=\frac{3 \cdot 83}{1 \cdot 41}=2 \cdot 71 \\
& \frac{4 \cdot 50}{\sqrt{3}}=\frac{4 \cdot 50}{1 \cdot 73}=2.60 \\
& \frac{5 \cdot 38}{\sqrt{4}}=\frac{5 \cdot 38}{2 \cdot 00}=2 \cdot 69 \\
& \frac{5 \cdot 99}{\sqrt{5}}=\frac{5 \cdot 99}{2 \cdot 23}=2 \cdot 69 \\
& \frac{6 \cdot 45}{\sqrt{6}}=\frac{6 \cdot 45}{2 \cdot 44}=2 \cdot 64 \\
& \frac{7 \cdot 16}{\sqrt{7}}=\frac{7 \cdot 16}{2 \cdot 64}=2.71 \\
& \frac{7 \cdot 56}{\sqrt{8}}=\frac{7 \cdot 56}{2 \cdot 82}=2 \cdot 68 \\
& \text { Mittel } 2 \cdot 69
\end{aligned}
$$

Es zeigt sich also, dass thatsächlich die gefundenen Werthe proportional der $\sqrt{v}$, dass also bei gleicher Temperaturdifferenz $\mathrm{z}$ wischen Abkühlungsobject und umgebender Luft die Wärmeabgabe im Wind proportional der Quadratwurzel aus der Wind geschwindigkeit zu setzen ist. Es ergiebt sich demnach auf diesem Wege das gleiche Resultat wie in den früheren Glaskugelversuchen, nur dass es nunmehr auch für längere Versuchsdauer und an einem Objecte von constanter Temperatur erwiesen ist. Eine grössere Genauigkeit konnte allerdings auch mit dieser Versuchsanordnung wegen der Inconstanz der Windgeschwindigkeit und der dadurch bewirkten Fehlerquellen nicht erzielt werden.

Die soeben ausgeführte Berechnung ist aber im Stande, uns einen noch klareren mathematischen Einblick in die hier waltende Gesetzmässigkeit zu verschaffen. Nennen wir die im Wind (von $v$ Metern pro Sec.) abgegebene Wärmemenge $W_{W}$, die in der Windstille abgegebene $W_{S t}$, so. würden unsere Versuchsergebnisse zu folgender Gleichung führen: 
oder

$$
\begin{aligned}
\frac{W_{W}}{W_{S t}} & =2 \cdot 69 \sqrt{v} \\
& =\sqrt{7.24} \cdot \sqrt{v}
\end{aligned}
$$

$$
\begin{aligned}
\left(\frac{W_{W}}{W_{S t}}\right)^{2} & =7.24 \cdot v \\
& =2 \times 3.62 \times v .
\end{aligned}
$$

Dies ist aber nichts anderes als der Ausdruck für eine reguläre Parabel vom Halbparameter 3.62. Ihr Brennpunkt muss also vom Scheitel 1.81 entfernt sein. Errichtet man zwei auf einander senkrechte Coordinaten, trägt auf der $y$-Axe als Ordinate die abgegebenen Wärmemengen in regelmässigen Abständen aufsteigend von 0 an auf, auf der $x$-Axe die Windstärken von 0 nach rechts aufsteigend in gleichen regelmässigen, je $1^{\mathrm{m}}$ pro Sec. entsprechenden Abständen und zeichnet die Parabel ein (s. Fig. 4), so bedeutet jeder Parabelpunkt dasjenige Vielfache von der Wärmeabgabe in der Windstille, welches unter dem Einfluss einer gewissen (durch Projection des Punkes auf die $x$ Axe aufindbaren) Windgeschwindigkeit eintritt und durch den zugehörigen Ordinatenwerth auf der $y$-Axe angezeigt wird. Man übersiehtdemnach mit Hülfe dieser

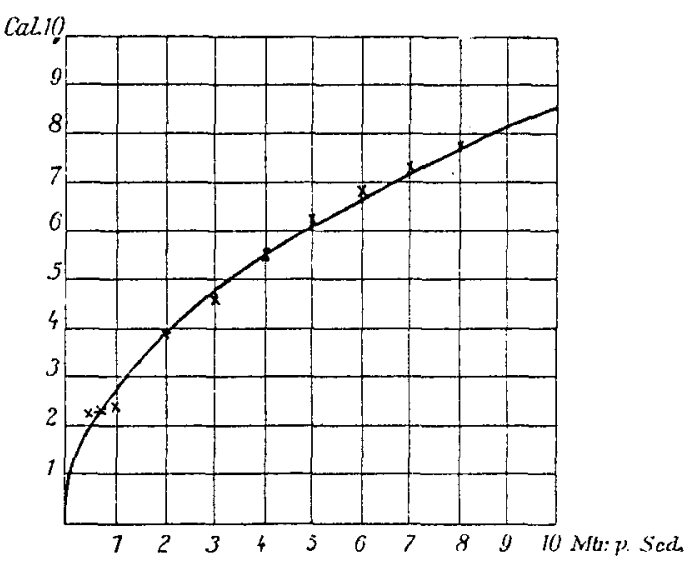

Fig. 4.

analytisch-geometrisehen Darstellung den Gang der Wärmeabgabe weit besser als durch die rein formale Angabe des Gesetzes. Besonders verständlich wird hierdurch auch die relativ viel stärkere Wirkung der schwächeren Winde gegenüber den stärkeren: Ein Blick auf die Eigenart der Parabelcurve macht es begreiflich, dass ein Wind von $0.5^{\mathrm{m}}$ pro Sec. die Wärmeabgabe schon um das 2 fache, ein Wind von $10 \mathrm{Mal}$ grösserer Geschwindigkeit, d. i. von $5^{\mathrm{m}}$ nur um das 6 fache, ein Wind von 20 Mal grösserer Geschwindigkeit, d. i. von $10^{\mathrm{m}}$ sogar nur um das $8 \frac{1}{2}$ fache steigert.

Es war nun zu prüfen, wie sich die im Versuch direct gefundenen Zahlen bezw. ihre relativen Werthe in diese Curve einfügten. Ich habe daher die Zahlen der letzten Horizontalcolumne aus Tabelle VIII an die entsprechende Stelle als kleine $\times$ in die Figur 4 eingetragen. In der That 
zeigt sich, dass diese aus den Versuchsergebnissen direct abgeleiteten Werthe zum Theil in dichter Nähe der Kurve liegen, zum Theil ihr geradezu angehören; die mathematische Reflexion steht demnach mit der experimentellen Beobachtung im Einklang: die im Wind beobachtete Erhöhung der Wärmeabgabe folgt dem Gange einer regulären Parabel.

Hat nun diese Thatsache nur für unsere besonderen Versuchsverhältnisse Gültigkeit, oder liegt hier eine allgemeine Gesetzmässigkeit ror? Man wird nicht fehlgehen, die letztere für die aufgefundenen Beziehungen in Anspruch zu nehmen. Wie aus dem F'rüheren hervorgeht, ist stets der Quotient $\frac{W_{W}}{W_{S t}}=$ einer constanten Zahl $c$ mal $\sqrt{v}$, und es muss immer möglich sein, den constanten Factor $c$, welchen Werth er auch haben möge, $=\sqrt{2 \cdot \frac{c^{2}}{2}}$ zu setzen und damit die Formel einer regulären Parabel aufzustellen.

Schwieriger ist die Frage, was die Constante $c$ bedeutet. Das Verständnis hierfür. wird erleichtert, wenn wir von den relativen Werthen nochmals zu den absoluten zurückkehren und der Formel eine allgemeinere Fassung geben. ${ }^{1}$ Wie dargethan, ist

also auch

$$
\frac{W_{W}}{W_{S t}}=c \sqrt{v}
$$

oder

$$
W_{W}=c \sqrt{v} \cdot W_{S t}
$$

$$
W_{W}=c \sqrt{v} \cdot(i+k) \tau T
$$

oder, wenn $\operatorname{man} c(i+k)=C$ setzt,

$$
W_{W}=C_{\tau} T \sqrt{v} .
$$

Es ist also die Constante $C$ abhängig von den Eigenschaften des abkühlenden Körpers, seiner Grösse, Form, Oberfläche und Substanz, sowie von seiner Aufstellung.

Die Formel $W_{W}=C \tau T \sqrt{v}$ ist der allgemeinste Ausdruck für die gefundenen Relationen; sie besagt, dass die Wärmeabgabe eines warmen Objectes in bewegter Luft direct proportional ist dem Product aus einer constanten Zahl, aus der Dauer der Entwärmung, aus der Temperaturdifferenz seiner Oberfläche mit der umgebenden Luft und aus der Quadratwurzel der Windgeschwindigkeit (Windgesetz).

${ }^{1}$ Für freundliche Unterstützung bei Ableitung der Formeln bin ich Hrn. Prof. Neumann za grossem Danke verpflichtet. 
Bei einem homogenen Körper, z. B. einer mit Wasser gefüllten Glaskugel, ist die Temperaturabnahme $\vartheta$ der Wärmeabgabe proportional, also auch der Differenz seiner Eigentemperatur mit der Lufttemperatur mal der Quadratwurzel aus der Windgeschwindigkeit proportional, d. h. es ist stets

$$
\vartheta=c T \sqrt{v}
$$

Legen wir nun eine bestimmte (die kleine Versuchs-) Glaskugel zu Grunde und setzen ihre Anfangstemperatur mit $30^{\circ}$ fest, so ist ihre Temperaturabnahme bei einer Lufttemperatur $t$ und einem Wind von $v$ Metern stets

$$
\vartheta=c(30-t) \sqrt{v}
$$

worin nur noch $c$ unbekannt ist. Dasselbe ist aus den Versuchsergebnissen leicht berechenbar. Ich habe die Berechnung für meine sämmtlichen Versuche ausgeführt und in Tabelle IX zusammengestellt.

Tabelle IX.

\begin{tabular}{r||c|c|c|c|c}
\hline \hline$v=$ & 23 & 30 & 38 & 51 & M i t t e l \\
\hline \hline$T=15$ & 0.097 & 0.088 & 0.088 & 0.097 & $=c=0.092$ \\
25 & 0.095 & 0.094 & 0.100 & 0.097 & $=c=0.096$ \\
35 & 0.106 & 0.095 & 0.088 & & $=c=0.096$ \\
& & & & \multicolumn{2}{c}{ Gesammit-Mittel $=0.094$}
\end{tabular}

Nennen wir den Temperaturabfall unserer Glaskugel bei einer Lufttemperatur $\boldsymbol{t}$ und einem Wind $v$ innerhalb 5 Minuten $\vartheta$ und setzen ihre Anfangstemperatur zu $30^{\circ}$ fest, so ist stets

$$
\vartheta=0.094(30-t) \sqrt{v} \text {. }
$$

Es ist also möglich, für jede beliebige Temperatur und jede Windgeschwindigkeit den Temperaturabfall der Kugel in Celsius-Graden an zugeben. Die resultierenden Werthe stellen die gemeinsame Wirkung der herrschenden Lufttemperatur und des Windes dar, es sind „combinirte" Ziffern, welche zur Charakteristik der jeweils herrschenden Witterung dienen können. Welche grosse Bedeutung dieselben für meteorologische und klimatologische Beobachtungen haben, wird an anderer Stelle ausführlich dargelegt werden. (Zur besseren Uebersicht und eventuell praktischen Verwendung habe ich in Tabelle $X$ die combinirten Ziffern bis zur Lufttemperatur $-5^{\circ}$ und bis zur Windgeschwindigkeit $15^{\mathrm{m}}$ pro Sec. zusammengestellt.) - 
Tabelle $\mathrm{X}$.

Abkühlung einer $30^{\circ}$ warmen Glaskugel um $\ldots{ }^{\circ} \mathrm{C}$. bei $t^{0}$ Lufttemperatur und $v$ Meter Wind innerhalb 5 Minuten.

\begin{tabular}{|c|c|c|c|c|c|c|c|c|c|c|c|c|c|c|c|}
\hline$v=$ & 1 & 2 & 3 & 4 & 5 & 6 & 7 & 8 & 9 & 10 & 11 & 12 & 13 & 14 & 15 \\
\hline$=29^{\circ}$ & $\| 0.09$ & $0 \cdot 13$ & $|0 \cdot 16|$ & $0 \cdot 19 \mid$ & 0.2 & $0.23 \mid$ & 0.25 & $0 \cdot 2$ & $0.28 \mid$ & $0 \cdot 30$ & 0.31 & 0.32 & $0 \cdot 34$ & 0.35 & $0 \cdot 36$ \\
\hline 28 & $0 \cdot 19$ & 0 . & $0.32\}$ & $0 \cdot 28$ & 0.42 & 0.46 & 0.50 & $0 \cdot 58$ & 0.56 & 0.59 & 0.62 & 0.65 & $0 \cdot 68$ & $0 \cdot 70$ & $0 \cdot 73$ \\
\hline 27 & 0.28 & 0.40 & $0.49 \mid$ & 56 & 0.63 & 0.69 & 0.74 & 0.79 & 0.85 & 0.89 & 0.93 & 0.98 & 1.01 & $1 \cdot 05$ & 1.09 \\
\hline 26 & $0 \cdot 38$ & 0.53 & $0.65 \mid$ & 5 & 0 & 0.92 & 99 & 1 & $|1 \cdot 13|$ & $1 \cdot 19$ & $1 \cdot 24$ & $1 \cdot 30$ & $1 \cdot 35$ & $1 \cdot 40$ & $1 \cdot 45$ \\
\hline 25 & 0.47 & 0.66 & 0.81 & 4 & 1 & $|1 \cdot 15|$ & 24 & 1 & $1 \cdot 41$ & $1 \cdot 49$ & 1.56 & $1 \cdot 63$ & $1 \cdot 69$ & $1 \cdot 76$ & $1 \cdot 82$ \\
\hline 24 & 0.56 & & 7 & 13 & $1 \cdot 25$ & $1 \cdot 37$ & $1 \cdot 49$ & & $1 \cdot 69$ & $1 \cdot 78$ & $1 \cdot 87$ & $1 \cdot 95$ & $2 \cdot 03$ & $2 \cdot 11$ & $2 \cdot 18$ \\
\hline 23 & 0.66 & 0.92 & $1 \cdot 13$ & 2 & 1 & 30 & 4 & 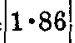 & 1.97 & $2 \cdot 08$ & $2 \cdot 18$ & $2 \cdot 28$ & $2 \cdot 37$ & $2 \cdot 46$ & $2 \cdot 54$ \\
\hline 22 & 0.75 & 06 & $|1 \cdot 30|$ & $1 \cdot 50$ & $1 \cdot 67$ & 83 & 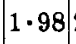 & 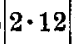 & $2 \cdot 26$ & $2 \cdot 38$ & $2 \cdot 49$ & $2 \cdot 60$ & $2 \cdot 70$ & $2 \cdot 81$ & $2 \cdot 90$ \\
\hline 21 & 0.85 & 19 & 1.46 & $1 \cdot 69$ & $1 \cdot 88$ & $2 \cdot 06$ & $|2 \cdot 23|$ & $2 \cdot 39$ & $2 \cdot 54$ & $2 \cdot 67$ & $2 \cdot 80$ & $2 \cdot 93$ & $3 \cdot 04$ & $3 \cdot 16$ & $3 \cdot 27$ \\
\hline 20 & 0.94 & $1 \cdot 32$ & 1.62 & $1 \cdot 88$ & $2 \cdot 09$ & $2 \cdot 29$ & $2 \cdot 48$ & $2 \cdot 65$ & $2 \cdot 82$ & $2 \cdot 97$ & $3 \cdot 11$ & $3 \cdot 25$ & $3 \cdot 38$ & $3 \cdot 51$ & $3 \cdot 63$ \\
\hline 19 & 1.03 & 45 & 1.78 & $2 \cdot 07$ & $2 \cdot 30$ & $2 \cdot 52$ & $2 \cdot 73$ & $2 \cdot 92$ & $3 \cdot 10 \mid$ & $3 \cdot 27$ & $3 \cdot 42$ & $3 \cdot 58$ & $3 \cdot 72$ & $3 \cdot 86$ & $3 \cdot 99$ \\
\hline 18 & $1 \cdot 13$ & 58 & 1.94 & $2 \cdot 26$ & $2 \cdot 51$ & $2 \cdot 75 \mid$ & $2 \cdot 98$ & $3 \cdot 19$ & $3 \cdot 38$ & $3 \cdot 56$ & $3 \cdot 73$ & $3 \cdot 90$ & $4 \cdot 06$ & $4 \cdot 21$ & $4 \cdot 46$ \\
\hline 17 & $1 \cdot 22$ & 72 & 2 & 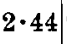 & $2 \cdot 72$ & $2 \cdot 98$ & $|3 \cdot 22|$ & $3 \cdot 45$ & $3 \cdot 67$ & $3 \cdot 86$ & $4 \cdot 04$ & $4 \cdot 23$ & $4 \cdot 39$ & $4 \cdot 56$ & $4 \cdot 72$ \\
\hline 16 & 32 & & 2 & $2 \cdot 63$ & $2 \cdot 93$ & 3 & 7 & 3 & $3 \cdot 95$ & $4 \cdot 16$ & $\cdot 35$ & $\cdot 55$ & $4 \cdot 73$ & $4 \cdot 91$ & $5 \cdot 08$ \\
\hline 15 & $1 \cdot 41$ & & & & & & 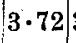 & 3 & $4 \cdot 23$ & $4 \cdot 46$ & 67 & 38 & .07 & $5 \cdot 27$ & .45 \\
\hline 14 & 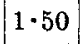 & & & & & & & & & $4 \cdot 75$ & $4 \cdot 98$ & $5 \cdot 20$ & $\cdot 41$ & $5 \cdot 62$ & 81 \\
\hline 13 & $1 \cdot 60$ & & & & & 3 & 2 & 4 & 79 & $5 \cdot 05$ & $\cdot 29$ & $5 \cdot 53$ & .75 & .97 & $\cdot 17$ \\
\hline 12 & 1.69 & & & & 3. & 4 & 4 & 4 & 08 & $5 \cdot 35$ & $5 \cdot 60$ & $5 \cdot 85$ & $6 \cdot 08$ & $6 \cdot 32$ & $\cdot 53$ \\
\hline 11 & 1.79 & 81 & & 3 & $3 \cdot 97$ & $4 \cdot 35$ & $4 \cdot 71$ & II & $5 \cdot 36$ & $5 \cdot 64$ & $5 \cdot 91$ & $6 \cdot 18$ & $6 \cdot 42$ & $6 \cdot 67$ & $6 \cdot 90$ \\
\hline 10 & $1 \cdot 88$ & 64 & $3 \cdot 24)$ & $3 \cdot 76$ & $4 \cdot 18$ & $|4 \cdot 58|$ & $\mid 4.96$ & $|5 \cdot 30|$ & $5 \cdot 64 \mid$ & $5 \cdot 94$ & $6 \cdot 22$ & $6 \cdot 50$ & $6 \cdot 76$ & $7 \cdot 02$ & $7 \cdot 26$ \\
\hline 9 & 1.97 & 77 & 03 & $3 \cdot 95$ & $4 \cdot 39$ & $4 \cdot 81$ & $|5 \cdot 21|$ & $5.57 \mid$ & $5 \cdot 92$ & $6 \cdot 24$ & $6 \cdot 53$ & $6 \cdot 83$ & $7 \cdot 10$ & $7 \cdot 37$ & $7 \cdot 62$ \\
\hline 8 & $2 \cdot 07$ & $2 \cdot 90$ & $3 \cdot 56$ & $4 \cdot 14$ & $4 \cdot 60$ & $5 \cdot 04$ & $5 \cdot 46$ & $5 \cdot 83 \mid$ & $6 \cdot 20 \mid$ & $6 \cdot 53$ & $6 \cdot 84$ & $7 \cdot 15$ & $7 \cdot 44$ & $7 \cdot 72$ & $7 \cdot 99$ \\
\hline 7 & $2 \cdot 16$ & 04 & $3 \cdot 73=$ & $4 \cdot 32$ & $4 \cdot 81$ & $5 \cdot 27$ & $5 \cdot 70 \mid$ & $6 \cdot 10$ & $6 \cdot 49$ & $6 \cdot 80$ & $7 \cdot 15$ & $7 \cdot 48$ & $7 \cdot 77$ & $8 \cdot 07$ & $8 \cdot 35$ \\
\hline 6 & $2 \cdot 26$ & 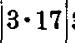 & 4 & 4 & $5 \cdot 02$ & $5.50\}^{5}$ & $5 \cdot 95$ & $6 \cdot 36 \mid$ & $6 \cdot 77$ & $7 \cdot 13$ & $7 \cdot 46$ & $7 \cdot 80$ & $8 \cdot 11$ & $8 \cdot 42$ & $8 \cdot 71$ \\
\hline 5 & $2 \cdot 35$ & 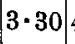 & 4 & 4 & $5 \cdot 23$ & $5 \cdot 736$ & 6. & 6 & $7 \cdot 05$ & $7 \cdot 43$ & $7 \cdot 78$ & $8 \cdot 13$ & $8 \cdot 45$ & $8 \cdot 78$ & $9 \cdot 08$ \\
\hline 4 & $2 \cdot 44$ & & & & $5 \cdot 43$ & $5 \cdot 95$ & $6 \cdot 45 \mid$ & $|0.00|$ & $7 \cdot 33$ & $7 \cdot 72$ & 8.09 & $8 \cdot 45$ & $8 \cdot 79$ & $9 \cdot 13$ & $9 \cdot 44$ \\
\hline 3 & $2 \cdot 54$ & 5 & & 8 & $5 \cdot 64$ & $6 \cdot 18$ & $6 \cdot 70 \mid 7$ & $7 \cdot 16 \mid$ & $7 \cdot 61$ & 8.02 & $8 \cdot 40$ & $8 \cdot 78$ & $9 \cdot 13$ & $9 \cdot 48$ & $9 \cdot 80$ \\
\hline 2 & $2 \cdot 63$ & & & $5 \cdot 26$ & $5 \cdot 85 \mid$ & $6 \cdot 41$ & 6.94 & $7 \cdot 42 \mid$ & $7 \cdot 90 \mid$ & $8 \cdot 32$ & $8 \cdot 71$ & $9 \cdot 10$ & $9 \cdot 46$ & $9 \cdot 83$ & 0.16 \\
\hline 1 & $2 \cdot 73 \mid$ & 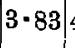 & & $5 \cdot 45$ & 6.06 & 6.64 & $7 \cdot 19$ & $7 \cdot 69$ & $8 \cdot 18$ & $8 \cdot 61$ & $9 \cdot 02$ & $9 \cdot 43$ & $9 \cdot 80$ & $10 \cdot 18$ & $10 \cdot 53$ \\
\hline 0 & $2 \cdot 82$ & 96 & $4 \cdot 86$ & 64 & $6 \cdot 27$ & $6 \cdot 877$ & $7 \cdot 44\}$ & $7 \cdot 95$ & $8 \cdot 46$ & $8 \cdot 91$ & $9 \cdot 33$ & $9 \cdot 75$ & $10 \cdot 14$ & $10 \cdot 53$ & $10 \cdot 89$ \\
\hline-1 & $2 \cdot 91$ & & & .83 & $6 \cdot 48$ & $7 \cdot 107$ & 7.69 & $8 \cdot 22$ & $8 \cdot 74$ & $9 \cdot 21$ & $9 \cdot 64$ & $10 \cdot 08$ & $10 \cdot 48$ & $10 \cdot 88$ & $11 \cdot 25$ \\
\hline-2 & $3 \cdot 01$ & $\cdot 22$ & 186 & $6 \cdot 0^{\prime}$ & $6 \cdot 69$ & $7 \cdot 337$ & $7 \cdot 948$ & $8 \cdot 48$ & $9 \cdot 02$ & $9 \cdot 50$ & $9 \cdot 95$ & $10 \cdot 40$ & $10 \cdot 82$ & $11 \cdot 23$ & $11 \cdot 62$ \\
\hline-3 & $3 \cdot 10$ & $\cdot 3$ & 5 & 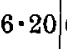 & $6 \cdot 90$ & $7 \cdot 5$ & $8 \cdot 1$ & $8 \cdot 75$ & $9 \cdot 31$ & $9 \cdot 80$ & $10 \cdot 26$ & $10 \cdot 73$ & $11 \cdot 15$ & $11 \cdot 58$ & $11 \cdot 98$ \\
\hline-4 & $3 \cdot 20$ & & & & & & 0 & $9 \cdot 01$ & $9 \cdot 59$ & $10 \cdot 10$ & 10.57 & $11 \cdot 05$ & $11 \cdot 49$ & $11 \cdot 93$ & $12 \cdot 34$ \\
\hline-5 & $3 \cdot 29$ & .62 & $5 \cdot 676$ & 0 & 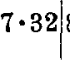 & $8 \cdot 0$ & $n^{2}$ & $9 \cdot 26$ & $9 \cdot 87$ & $10 \cdot 40$ & $10 \cdot 89$ & 88 & $11 \cdot 83$ & $12 \cdot$ & $12 \cdot 71$ \\
\hline
\end{tabular}


Ebenso sei hier nur kurz darauf hingewiesen, dass sich an der Hand des Windgesetzes auch die Wirkung der stossweisen Winde, welche ja gerade im Freien die grösste Rolle spielen, in einfacher und übersichtlicher Weise zur Darstellung bringen lässt. -

Es erübrigt nun noch, die Gültigkeit des Gesetzes anch für die Grenzwerthe von $v$ zu untersuchen. Gehen wir wiederum von der Formel

$$
\frac{W_{W}}{W_{S t}}=c \sqrt{v}=\sqrt{c^{2} v}
$$

aus; wird die Windgeschwindigkeit $v$ immer kleiner, so wird sie zunächst den reciproken Werth von $c^{2}$ erreichen, also

sein, also

$$
\frac{W_{W}}{W_{S t}}=\sqrt{c^{2} \cdot \frac{1}{c^{2}}}
$$

oder

$$
\frac{W_{W}}{W_{S t}}=1
$$

$$
W_{W}=W_{S t} .
$$

Es würde also unter unseren Versuchsbedingungen bei einem Wind von $\frac{1}{7 \cdot 24}=0.13^{\mathrm{m}}$ pro Sec., mithin bei relativ starkem Wind, nur dieselbe Wärmeabgabe stattfinden, wie in Windstille. Die Formel würde also für alle der 0 benachbarten Werthe von $v$ ungültig sein. Dieser Widerspruch ist jedoch lösbar; er beruht darauf, dass der in den Tabellen als „Windstille" bezeichnete Zustand der Luft kein id ealer Beharrungszustand ist, keine mathematische Windstille, in welcher $v=0 \mathrm{zu}$ setzen ist, sondern eine, wenn auch geringe, so doch unter natürlichen Verhältnissen durch Heizung, Ventilation, Erschütterungen u. s. w. stets vorhandene Bewegung der Luft, welche nur im Gegensatz zu den starken, mit Hülfe des Ventilators erzeugten luftströmen als Windstille aufgefasst werden konnte. Diese natürliche Luftbewegung muss aber noch eine besondere Steigerung in der Nähe des warmen Objectes erfahren, längs dessen je nach Temperaturdifferenz, Lage und Form ein mehr oder minder lebhafter Ventilationsstrom hinstreichen wird und in unserem besonderen Falle mit durchschnittlich $13^{\mathrm{cm}}$ pro Sec. vielleicht nicht zu hoch gefunden ist. Setzen wir statt des, in den Tabellen als Windstille bezeichneten, Zustandes der Luft die eben genannte Luftbewegung ein, so ist die Formel anch für sehr kleine Werthe ron $v$ brauchbar, ja selbst bis an die äussersten Grenzwerthe einer Deutung zugänglich. Wird nämlich $v=0$, so wird - in der Scheitelgleichung der Parabel - auch $y=0, \mathrm{~d}$. h. der gesuchte Werth liegt im Schnittpunkt der $x$ - und $y$-Axe. Unter realen Verhältnissen ist $v=0$ nur denkbar, wenn sich das Object im luftleeren Raume oder in 
228 Bruno Heymann: Ennfluss D. Windess a. D. Wärmedbgabe d. s. W.

Luft von einer, mit der Oberflächentemperatur des Körpers übereinstimmenden Temperatur befindet, also unter Verhältnissen, wo die Wärmeabgabe durch Leitung überhaupt ausgeschlossen ist. - Wird hingegen die Windgeschwindigkeit $v$ immer grösser, so flacht sich die Curve mehr und mehr ab, bis sie schliesslich bei $v=\infty$ der Abscisse parallel läuft. Interessanter Weise existiren zwei real denkbare Möglichkeiten, wo selbst eine solche, unendlich starke Bewegung des Mediums keine Multiplikation der Wärmeabgabe zu Stande bringt, nämlich wiederum im luftleeren Raum und im gleichwarmen Luftbade, also wiederum unter Bedingungen völlig behinderter Wärmeleitung.

Schliesslich will ich noch der bemerkenswerthen Thatsache Erwähnung thun, dass Schierbeck ${ }^{1}$ die austrocknende Wirkung des Windes gleichfalls proportional der Quadratwurzel aus seiner Geschwindigkeit gefunden hat, eine Analogie, welche bei der Aehnlichkeit des Verdunstungs- und Abkühlungsprocesses gewiss im Sinne einer Bestätigung des Windgesetzes verwerthet werden darf. -

Die Verfolgung des Windeinflusses auch auf lebende Wesen, insbesondere auf den Menschen, ist weiteren Versuchen vorbehalten, mit deren Ausführung ich beschäftigt bin.

1 Schierbeck, Ueber die Bestimmung des Feuchtigkeitsgrades der Luft für physiologische und hygienische Zwecke. Archiv für Bygiene. 1895. Bd. XXV. S. 196. 